\title{
The complemented subspace problem revisited
}

by

\author{
N. J. Kalton (Columbia, MO)
}

\begin{abstract}
We show that if $X$ is an infinite-dimensional Banach space in which every finite-dimensional subspace is $\lambda$-complemented with $\lambda \leq 2$ then $X$ is $(1+C \sqrt{\lambda-1})$-isomorphic to a Hilbert space, where $C$ is an absolute constant; this estimate (up to the constant $C$ ) is best possible. This answers a question of Kadets and Mityagin from 1973. We also investigate the finite-dimensional versions of the theorem.
\end{abstract}

1. Introduction. In 1971, Lindenstrauss and Tzafriri [13] proved that a Banach space in which every closed subspace is the range of a bounded projection is (isomorphically) a Hilbert space. This theorem is based on a quantitative result. They show that if $X$ is an infinite-dimensional Banach space with the property that every finite-dimensional subspace is $\lambda$-complemented then $d_{X} \leq 2^{9} \lambda^{4}$ where $d_{X}=d(X, H)$ for $H$ a Hilbert space of the same density character as $X$.

Let us introduce the following notation. If $X$ is any Banach space let $\lambda_{X}$ be the least constant such that every finite-dimensional subspace of $X$ is $\lambda_{X}$-complemented. Let $\varphi(\lambda)$ be the optimal function such that $d_{X} \leq \varphi\left(\lambda_{X}\right)$ for every infinite-dimensional real Banach space $X$ for which $\lambda_{X}<\infty$ (it is clear that such an optimal function exists). Thus Lindenstrauss and Tzafriri showed that $\varphi(\lambda) \leq 2^{9} \lambda^{4}$. Later this was improved by Kadets and Mityagin [10] to $\varphi(\lambda) \leq 8 \lambda^{2}$ and then by Figiel [6] to $\varphi(\lambda) \leq 4 \lambda^{2}$. For an exposition see [1].

On the other hand, in 1939 Kakutani [11], extending an earlier result of Blaschke from 1916 [3], proved that for any real Banach space of dimension at least 3, if every two-dimensional subspace is 1-complemented then $X$ is isometric to a Hilbert space. In particular, $\varphi(1)=1$. This led Kadets and Mityagin to ask whether a proof could be given which unified the BlaschkeKakutani theorem with the Lindenstrauss-Tzafriri theorem; essentially this

2000 Mathematics Subject Classification: Primary 46B03, 46B20.

Key words and phrases: complemented subspace, perturbation of Hilbert space, Dvoretzky theorem, Hilbert enlargement, reflection.

The author was supported by NSF grants DMS-0244515 and DMS-0555670. 
asks whether $\varphi$ is continuous at $\lambda=1$. In particular, they asked whether the factor 8 (or in Figiel's version 4) could be removed, i.e. whether $\varphi(\lambda) \leq \lambda^{2}$.

Our main result is the following (see Theorem 6.2 below and the subsequent examples):

TheOREm 1.1. There exist constants $0<c<C<\infty$ so that

$$
1+c \sqrt{\lambda-1} \leq \varphi(\lambda) \leq 1+C \sqrt{\lambda-1}, \quad 1 \leq \lambda \leq 2 .
$$

This shows that $\varphi$ is continuous at 1 but that the conjecture that $\varphi(\lambda) \leq \lambda^{2}$ is false.

Curiously, the main element in the proof is a similar estimate for a very special type of three-dimensional space (see Theorems 5.1 and 5.7). Unfortunately, the proof of Theorem 5.1 is rather long and somewhat tedious.

It is also possible to consider the finite-dimensional versions of these results. We do not know if the conclusion of Theorem 1.1 holds for finitedimensional spaces. However, we are able to prove at least some partial results.

For each $n \geq 3$ we define $\varphi_{n}(\lambda)$ to be the least constant so that if $\operatorname{dim} X=n$ and every subspace is $\lambda$-complemented then $d_{X} \leq \varphi_{n}\left(\lambda_{X}\right)$. We can then define $\varphi_{\infty}(\lambda)=\sup _{n>3} \varphi_{n}(\lambda)$. Figiel, Lindenstrauss and Milman [7] proved that $\varphi_{\infty}(\lambda) \leq C \lambda^{32}$ for some constant $C$. The following is clear from the compactness of the Banach-Mazur compactum:

Proposition 1.2. For each $n \geq 3$,

$$
\lim _{\lambda \rightarrow 1} \varphi_{n}(\lambda)=1 \text {. }
$$

We are able to give an estimate of $\varphi_{n}(\lambda)$ which is slightly weaker than the conclusion of Theorem 1.1 (see Theorem 8.1 below):

Theorem 1.3. Suppose $n \in \mathbb{N}$ and $0<a<1 / 3$. Then there exists a constant $0<C<\infty$ so that

$$
\varphi_{n}(\lambda) \leq 1+C\left(\sqrt{\lambda-1}+n^{-a}\right), \quad 1 \leq \lambda \leq 2 .
$$

However, it is not obvious that $\varphi_{\infty}$ is continuous at 1 . This is our second main result (Theorem 8.4):

TheOREM 1.4. We have

$$
\lim _{\lambda \rightarrow 1} \varphi_{\infty}(\lambda)=1 .
$$

Unfortunately, we do not give an explicit estimate for $\varphi_{\infty}(\lambda)$ as our results depend on the unknown form of $\varphi_{3}$.

In the complex case the analogue of Kakutani's theorem is due to Bohnenblust [4]. Thus the same questions may be asked for complex Banach spaces. There is however a priori reason to expect the complex analogues of the functions $\varphi, \varphi_{n}, \varphi_{\infty}$ should coincide with the real functions. Nevertheless, 
all our results above are valid for the similarly defined complex analogues of these functions.

We obtain Theorem 1.4 by consideration of a second problem. A symmetry on a real or complex Banach space is an operator $S$ such that $S^{2}=I$. Then $S=I-2 P$, where $P$ is a projection whose kernel ker $P$ is the fixed subspace of $S$, i.e. $\{x: S x=x\}$, and whose range is the fixed subspace of $-S$. If $X$ is a Banach space, we may define the symmetry constant $\sigma_{X}$ to be the least constant so that for every finite-dimensional subspace $E$ there is a symmetry $S$ whose fixed subspace is $E$ and $\|S\| \leq \sigma_{X}$. Equivalently, there is a projection $P$ of $X$ onto $E$ with $\|I-2 P\| \leq \sigma_{X}$. Clearly, $1 \leq \lambda_{X} \leq \sigma_{X} \leq d_{X}$. For $\sigma_{X}$ we can give a rather more precise result (Theorems 7.9 and 8.2):

TheOREM 1.5. There exists a constant $1 \leq C<\infty$ so that if $X$ is an infinite-dimensional real or complex Banach space with $\sigma_{X}<2$ then

$$
\sigma_{X}-1 \leq d_{X}-1 \leq C\left(\sigma_{X}-1\right) .
$$

TheOREM 1.6. There is a constant $C$ so that if $X$ is a finite-dimensional Banach space with $\sigma_{X}<2$ then

$$
d_{X}-1 \leq C\left(\sigma_{X}-1\right)\left|\log \left(\sigma_{X}-1\right)\right| .
$$

We also introduce the reflection constant $\varrho_{X}$, which we define to be the least constant $\varrho$ such that for every one-dimensional subspace $E$ of $X$ there is a symmetry $S: X \rightarrow X$ such that $E=\{x: S x=x\}$ and $\|S\| \leq \varrho$. We show:

TheOREM 1.7. There is a constant $C$ so that if $X$ is an arbitrary Banach space we have

$$
\varrho_{X}-1 \leq d_{2}(X)-1 \leq C\left(\varrho_{X}-1\right)
$$

where

$$
d_{2}(X)=\sup \left\{d_{E}: E \text { is a subspace of } X \text { with } \operatorname{dim} E=2\right\} .
$$

The proof of this result is relatively easy for real spaces but is rather cumbersome in the complex case. The difficulties arise for a two-dimensional complex space; we feel that our method is not optimal and there should be a simpler argument.

We would like to thank Olek Pełczyński for drawing our attention to this problem and for many helpful comments. We also acknowledge useful discussions with Joram Lindenstrauss. We would also like to thank the referee for a very careful reading of the paper, which led to many corrections and improvements in the argument.

2. Preliminaries. In this section we introduce a number of definitions and basic results which will be needed in the rest of the paper. For a Banach space $X$ we use the notation $B_{X}=\{x:\|x\| \leq 1\}$ and $\partial B_{X}=\{x:\|x\|=1\}$. 
We recall that if $X$ and $Y$ are Banach spaces, the Banach-Mazur distance $d(X, Y)$ is defined by

$$
d(X, Y)=\inf \left\{\|T\|\left\|T^{-1}\right\|: T: X \rightarrow Y \text { is an isomorphism }\right\}
$$

with $d(X, Y)=\infty$ for non-isomorphic Banach spaces. We define

$$
d_{X}=\inf \{d(X, H): H \text { is a Hilbert space }\} .
$$

We also introduce the constants $\lambda_{X}, \sigma_{X}$ and $\varrho_{X}$. We let $\lambda_{X}$ be the infimum of all $\lambda$ such that for every finite-dimensional subspace $E$ of $X$, there is a projection $P: X \rightarrow E$ with $\|P\| \leq \lambda$. If $\lambda_{X}<\infty$, i.e. all finite-dimensional subspaces are uniformly complemented, then it may easily be seen by a compactness argument that there is a projection $P: X \rightarrow E$ with $\|P\| \leq \lambda_{X}$. Thus the definition may be stated as in the Introduction.

A symmetry on $X$ is a linear operator $S$ with $S^{2}=I$. We let the symmetry constant $\sigma_{X}$ be the infimum of all $\sigma$ such that for every finite-dimensional subspace $E$ of $X$, there is a symmetry $S: X \rightarrow X$ with $E=\{x: S x=x\}$ and $\|S\| \leq \sigma$. As before, if $\sigma_{X}<\infty$ then we can find a symmetry fixing $E$ with $\|S\| \leq \sigma_{X}$. A reflection on $X$ is a symmetry $R$ such that $\{x: R x=x\}$ has dimension one. We let the reflection constant $\varrho_{X}$ be the infimum of all $\varrho$ such that for every one-dimensional subspace $E$ of $X$, there is a reflection $R: X \rightarrow X$ with $E=\{x: R x=x\}$ and $\|R\| \leq \varrho$. If $\varrho_{X}<\infty$ then we can find a reflection fixing $E$ with $\|R\| \leq \varrho_{X}$.

Let us first record the Lindenstrauss-Tzafriri theorem [13] in the form we will need (for finite dimensions it is due to Figiel, Lindenstrauss and Milman [7]):

THEOREM 2.1. If $X$ is a Banach space and $a>1$, there is a constant $M=M(a)$ so that $\lambda_{X} \leq a$ implies $d_{X} \leq M$.

If $X$ is a Banach space, we define

$$
d_{m}(X)=\sup \left\{d_{E}: E \subset X, \operatorname{dim} E=m\right\} .
$$

The following result is due to Figiel, Lindenstrauss and Milman [7, Theorem 6.2]:

Proposition 2.2. If $X$ is an $n$-dimensional Banach space and $2 \leq k \leq n$ then

$$
\log d_{X} \leq 2\left(1+\frac{\log n}{\log k}\right) \log d_{k}(X)
$$

We shall need the following:

Proposition 2.3. Suppose $1<a<\sqrt{2}$. Then there exists $p=p(a)>1$ and a constant $C=C(a)$ so that if $X$ is a Banach space with $d_{2}(X) \leq a$ then $X$ has Rademacher type $p$ with type $p$ constant $T_{p}(X) \leq C$. 
Proof. If this is false then we can find, for some $1<a<\sqrt{2}$, a sequence of Banach spaces $X_{n}$ so that $d_{2}\left(X_{n}\right) \leq a$ but $T_{1+1 / n}\left(X_{n}\right) \geq n$. Then the $\ell_{2}$-sum $Y=\ell_{2}\left(X_{n}\right)$ fails to have any non-trivial type. However, it follows from the fact that each $X_{n}$ satisfies $d_{2}\left(X_{n}\right) \leq a$ that we have an estimate

$$
\left\|y_{1}+y_{2}\right\|^{2}+\left\|y_{1}-y_{2}\right\|^{2} \leq 2 a^{2}\left(\left\|y_{1}\right\|^{2}+\left\|y_{2}\right\|^{2}\right), \quad y_{1}, y_{2} \in Y .
$$

In particular, if $\left\|y_{1}\right\|=\left\|y_{2}\right\|=1$ then either $\left\|y_{1}-y_{2}\right\|<a \sqrt{2}$ or $\left\|y_{1}+y_{2}\right\|<$ $a \sqrt{2}$. However, $\ell_{1}$ is finitely representable in $Y$ (see [1, p. 270] for example).

We denote the closed unit ball of a Banach space $X$ by $B_{X}$ and the unit sphere by $\partial B_{X}$.

Proposition 2.4. Suppose $X$ is a Banach space.

(i) Suppose that for every closed subspace $E$ of codimension one there is a projection $P: X \rightarrow E$ with $\|P\| \leq \tau$. If $\tau<\mu<\infty$ then there is a continuous map $\Phi: \partial B_{X^{*}} \rightarrow \mathcal{L}(X)$ so that $\Phi\left(x^{*}\right)=\Phi\left(\alpha x^{*}\right)$ if $|\alpha|=1$, and for each $x^{*} \in \partial B_{X^{*}}, \Phi\left(x^{*}\right)$ is a projection onto ker $x^{*}$ with $\left\|\Phi\left(x^{*}\right)\right\|<\mu$.

(ii) Suppose that for every subspace $E$ of dimension one there is a projection $P: X \rightarrow E$ with $\|I-2 P\| \leq \tau$. If $\tau<\mu<\infty$ then there is a continuous map $\Phi: \partial B_{X} \rightarrow \mathcal{L}(X)$ so that $\Phi(\alpha x)=\Phi(x)$ if $|\alpha|=1$, and for each $x \in \partial B_{X}, \Phi(x)$ is a projection onto $[x]$ with $\|I-2 \Phi(x)\|<\mu$.

Proof. Both parts follow from the Michael selection theorem (see [2, p. 22]).

(i) Fix $\tau<\beta<\mu$. For each $x^{*} \in \partial B_{X^{*}}$ define $K\left(x^{*}\right)$ to be the set of projections $P: X \rightarrow \operatorname{ker} x^{*}$ with $\|P\| \leq \beta$. Then $K\left(x^{*}\right)$ is non-empty, closed and convex. We show that $K$ is lower-semicontinuous. Indeed, suppose $\mathcal{U}$ is an open subset of $\mathcal{L}(X)$ and that $K\left(x^{*}\right) \cap \mathcal{U} \neq \emptyset$. Then there exists $P \in K\left(x^{*}\right) \cap \mathcal{U}$ with $\|P\|<\beta$. Suppose $y^{*} \in \partial B_{X^{*}}$ and $\left\|y^{*}-x^{*}\right\|<\nu$ where $\|P\|(1+\nu)(1-\nu)^{-1} \leq \beta$. Pick $x \in X$ with $\|x\|=1=x^{*}(x)$ and consider the map $A: X \rightarrow X$ defined by $A z=\left(x^{*}-y^{*}\right)(z) x$ (we recall that $X$ is isomorphic to a Hilbert space and hence reflexive). Then $I-A$ is an automorphism of $X$ which maps $\operatorname{ker} x^{*}$ onto ker $y^{*}$. Hence $(I-A) P(I-A)^{-1}$ is a projection onto $\operatorname{ker} y^{*}$ and

$$
\left\|(I-A) P(I-A)^{-1}\right\| \leq \frac{1+\nu}{1-\nu}\|P\| .
$$

Therefore $K\left(y^{*}\right) \cap \mathcal{U} \neq \emptyset$ and $K$ is lower-semicontinuous. By the Michael selection theorem we have the existence of a continuous selection $\Phi_{0}\left(x^{*}\right) \in$ 
$K\left(x^{*}\right)$. We can then define

$$
\Phi\left(x^{*}\right)= \begin{cases}\frac{1}{2}\left(\Phi_{0}\left(x^{*}\right)+\Phi_{0}\left(-x^{*}\right)\right) & \text { in the real case, } \\ \frac{1}{2 \pi} \int_{0}^{2 \pi} \Phi_{0}\left(e^{i \theta} x^{*}\right) d \theta & \text { in the complex case. }\end{cases}
$$

(ii) The proof is very similar and we omit the details.

3. Perturbations of Hilbert spaces. Let $H$ be a real or complex Hilbert space; we denote the norm by $|\cdot|$. We will consider an equivalent norm $\|\cdot\|$ on $H$. For $0<\delta<1$ we will say that $\|\cdot\|$ is a $\delta$-perturbation of $|\cdot|$ if the function $f(x)=\|x\|-|x|$ has Lipschitz constant $\operatorname{Lip}(f) \leq \delta$ with respect to $(H,|\cdot|)$.

If we denote by $X$ the Banach space $(H,\|\cdot\|)$ then we will also say that $X$ is a $\delta$-perturbation of $H$.

If $\|\cdot\|$ is any equivalent norm on $H$ then we can define the dual norm $\|\cdot\|_{*}$ via the duality, i.e.

$$
\|x\|_{*}=\sup \left\{|(x, y)|:\|y\|_{X} \leq 1\right\} .
$$

If $T$ is a bounded operator on $H$ then we denote by $|T|$ its norm and $\|T\|$ its norm on $(H,\|\cdot\|)$.

The following lemma is trivial:

LEMma 3.1. If $\|\cdot\|$ is a $\delta$-perturbation of $|\cdot|$ then

$$
(1-\delta)|x| \leq\|x\| \leq(1+\delta)|x|, \quad x \in H,
$$

and so

$$
d_{X} \leq \frac{1+\delta}{1-\delta} .
$$

We shall be interested in giving conditions where a converse to this lemma holds.

Lemma 3.2. Assume that $\|\cdot\|$ is a norm on $H$. Suppose that $0<\delta, \tau, \nu<1$ are such that

$$
(1-\delta)|x| \leq\|x\| \leq(1+\delta)|x|, \quad x \in H,
$$

and

$$
\left|x-x^{\prime}\right| \leq \nu,|x|=\left|x^{\prime}\right|=1 \Rightarrow\left|\|x\|-\left\|x^{\prime}\right\|\right| \leq \tau .
$$

Then $\|\cdot\|$ is $a(\delta+\nu+\nu \tau / 2+\tau / \nu)$-perturbation of $|\cdot|$.

In particular, if $\|x\|$ has Lipschitz constant $\kappa$ on the set $\{x:|x|=1\}$ then $\|x\|$ is a $(\delta+\kappa)$-perturbation of $|\cdot|$.

Proof. Let $f(x)=\|x\|-|x|$. We can estimate the Lipschitz constant of $f$ by considering its (almost everywhere defined) gradient on any finitedimensional subspace. Suppose therefore $H$ is finite-dimensional. Suppose 
$|x|=1$ and $x$ is a point of differentiability of $f$. Then $\nabla f=x^{*}-x$ where $x^{*} \in H$ satisfies $\left(x, x^{*}\right)=\|x\|$ and $\left\|x^{*}\right\|_{*}=1$. Let $x^{*}=\|x\| x+u$ where $(x, u)=0$. Then for $|w| \leq 1$ and $(w, x)=0$

$$
\begin{aligned}
\left(x+\nu w, x^{*}\right) & =\|x\|+\nu(w, u) \leq\|x+\nu w\| \leq\left(1+\nu^{2}\right)^{1 / 2}(\|x\|+\tau) \\
& \leq\|x\|+\tau+\frac{1}{2} \nu^{2}(1+\delta+\tau) .
\end{aligned}
$$

Hence $\nu(w, u) \leq \tau+\frac{1}{2} \nu^{2}(1+\delta+\tau)$ and so $|u| \leq \tau / \nu+\frac{1}{2} \nu(1+\delta+\tau)$ and

$$
|\nabla f| \leq\left((\|x\|-1)^{2}+|u|^{2}\right)^{1 / 2} \leq \delta+\nu+\nu \tau / 2+\tau / \nu .
$$

This estimate on $|\nabla f|$ proves the result.

For the second part we observe that the estimate holds with $\tau=\kappa \nu$ for any $\nu>0$.

Proposition 3.3. Suppose $0<\delta<1$ and $\|\cdot\|$ satisfies

$$
(1-\delta)|x| \leq\|x\| \leq(1+\delta)|x|, \quad x \in H .
$$

Then $\|\cdot\|$ is a $5 \sqrt{\delta}$-perturbation of $|\cdot|$.

Proof. Let $\nu=\sqrt{\delta}$ in the preceding Lemma 3.2 and let $\tau=2 \delta$. The result is immediate.

In fact, Proposition 3.3 is sharp (up to the constant) and we will demonstrate this in the next section. However, one can improve on that proposition under some very special hypotheses.

Proposition 3.4. Suppose $0<\delta<1 / 2$ and $\|\cdot\|$ satisfies

$$
(1-\delta)|x| \leq\|x\| \leq(1+\delta)|x|, \quad x \in H .
$$

Suppose additionally that for some $n \in \mathbb{N},\|x\|^{2 n}$ is a polynomial of degree $2 n$ (for instance, if $(H,\|\cdot\|)$ embeds isometrically into $L_{2 n}$ ). Then $\|\cdot\|$ is a $\left(3^{2 n-1} \pi n+1\right) \delta$-perturbation of $|\cdot|$.

Proof. Let $V$ be a two-dimensional subspace of $H$ with orthonormal basis $\{u, v\}$. Let $\varphi(t)=\|u \cos t+v \sin t\|$. Then $\varphi(t)^{2 n}$ is a trigonometric polynomial of degree $2 n$ so that by Bernstein's inequality (see Zygmund [20, Vol. II, p. 11]),

$2 n \varphi(t)^{2 n-1}\left|\varphi^{\prime}(t)\right| \leq 2 n \max _{0 \leq s \leq 2 \pi}\left|\varphi(s)^{2 n}-1\right| \leq 2 n\left((1+\delta)^{2 n}-1\right), \quad 0 \leq t \leq 2 \pi$.

Hence

$$
\left|\varphi^{\prime}(t)\right| \leq \frac{(1+\delta)^{2 n}-1}{(1-\delta)^{2 n-1}}, \quad 0 \leq t \leq 2 \pi
$$

Note that

$$
(1+\delta)^{2 n}-1 \leq 2 n \delta(1+\delta)^{2 n-1}
$$

so that we have

$$
\left|\varphi^{\prime}(t)\right| \leq 2 \cdot 3^{2 n-1} n \delta, \quad 0 \leq t \leq 2 \pi
$$


This implies that if $x, x^{\prime} \in V$ with $|x|=\left|x^{\prime}\right|=1$ then

$$
\left|\|x\|-\left\|x^{\prime}\right\|\right| \leq 3^{2 n-1} \pi n \delta\left|x-x^{\prime}\right| .
$$

Applying Lemma 3.2 shows that $\|\cdot\|$ is a $\left(3^{2 n-1} \pi n+1\right) \delta$-perturbation of $|\cdot|$ on $V$; since this holds for every 2-dimensional subspace the proof is complete.

The following lemma will be a key ingredient of our proof of the main theorem.

Lemma 3.5. Let $\|\cdot\|$ be a $\delta$-perturbation of $|\cdot|$ where $0<\delta<1 / 3$. Suppose $|x|=|y|=1$.

(i) If $(x, y)=0$ then

$$
\|x\| \leq\left(1+\delta^{2}\right)\|x+t y\|, \quad-\infty<t<\infty .
$$

(ii) If $0<\nu<1$ is such that

$$
\|x\| \leq(1+\nu)\|x+t y\|, \quad-\infty<t<\infty,
$$

then

$$
|(x, y)| \leq \delta+\sqrt{2 \nu}
$$

Proof. (i) Let $f(x)=\|x\|-|x|$. Then

$$
\begin{aligned}
\|x+t y\|-\|x\| & =|x+t y|-|x|+f(x+t y)-f(x) \\
& \geq\left(1+t^{2}\right)^{1 / 2}-1-\delta|t|, \quad-\infty<t<\infty .
\end{aligned}
$$

The minimum of the right-hand side occurs when $t=\delta\left(1-\delta^{2}\right)^{-1 / 2}$, and so

$$
\|x+t y\|-\|x\| \geq\left(1-\delta^{2}\right)^{1 / 2}-1 \geq-\delta^{2} / 2, \quad-\infty<t<\infty .
$$

Since $\delta<1 / 3$ we have $\|x\| \geq 2 / 3$. It follows that

$$
\|x+t y\| \geq\left(1-\frac{3}{4} \delta^{2}\right)\|x\|, \quad-\infty<t<\infty .
$$

Thus

$$
\|x\| \leq\left(1-\frac{3}{4} \delta^{2}\right)^{-1}\|x+t y\| \leq\left(1+\delta^{2}\right)\|x+t y\|, \quad-\infty<t<\infty .
$$

(ii) In this case we have

$$
\begin{aligned}
|x+t y|-1 & =\|x+t y\|-\|x\|-(f(x+t y)-f(x)) \\
& \geq-\nu\|x\|-|t| \delta, \quad-\infty<t<\infty .
\end{aligned}
$$

Hence

i.e.

$$
|x+t y|^{2} \geq(1-2 \nu-|t| \delta)^{2}, \quad-\infty<t<\infty,
$$

$$
t^{2}+2 t(x, y)+1 \geq(1-2 \nu)^{2}-2|t|(1-2 \nu) \delta+t^{2} \delta^{2}, \quad-\infty<t<\infty .
$$

We can clearly reduce considerations to the case when $t(x, y) \leq 0$ and so the quadratic

$$
\left(1-\delta^{2}\right) t^{2}+2 t((x, y)-\operatorname{sgn}(x, y)(1-2 \nu) \delta)+2 \nu-\nu^{2}
$$


is always non-negative. Hence so is the quadratic $t^{2}+2 t(|(x, y)|-\delta)+2 \nu$. Checking the discriminant, if $|(x, y)| \geq \delta$, we get $|(x, y)|-\delta \leq \sqrt{2 \nu}$.

Theorem 3.6. Suppose $\|\cdot\|$ is a $\delta$-perturbation of $|\cdot|$, where $\delta<1 / 3$. Then, if $P$ is an orthogonal projection on $(H,|\cdot|)$, we have $\|P\| \leq 1+\delta^{2}$.

Proof. This follows directly from the previous lemma.

4. Dvoretzky's theorem for small perturbations. In this section, we point out some simple consequences of the concentration of measure phenomenon for small perturbations of Hilbert spaces.

TheOREM 4.1. Let $X$ be an $n$-dimensional normed space. Suppose that $m \in \mathbb{N}$ satisfies $4<m \leq \min \left(\frac{1}{2} n-2,25\left(d_{X}-1\right) n\right)$ and

$$
48 d_{X} \sqrt{\frac{m}{n} \log \frac{n}{m}}<1 .
$$

Let $E$ be an $m$-dimensional subspace of $X$. Then there is a subspace $F$ of $X$ with $E \cap F=\{0\}$ and such that there is a Euclidean norm $|\cdot|$ on $F$ so that if $U: F \rightarrow F$ is an isometry with respect to $|\cdot|$ then the operator $\widetilde{U}: E+F \rightarrow E+F$ defined by

$$
\widetilde{U}(e+f)=e+U f, \quad e \in E, f \in F,
$$

satisfies

$$
\|\widetilde{U}\| \leq \begin{cases}1+40 d_{X} \sqrt{\frac{m}{n} \log \frac{n}{m}}, & d_{X}>1.04, \\ 1+200 \sqrt{d_{X}-1} \sqrt{\frac{m}{n} \log \frac{n}{m}}, & d_{X} \leq 1.04 .\end{cases}
$$

Proof. We give the proof for real scalars. We may assume the existence of a Euclidean norm $|\cdot|$ on $X$ so that $d_{X}^{-1}|x| \leq\|x\| \leq|x|$ for $x \in X$. We will work with respect to this inner product. Let $\theta=\min \left(1,5 \sqrt{d_{X}-1}\right)$. Note that

$$
\frac{m}{n} \leq \min \left(\frac{1}{2}, 25\left(d_{X}-1\right)\right) \leq \theta^{2} .
$$

We then fix

$$
\nu=3 \sqrt{\frac{m}{n} \log \frac{n}{m}} .
$$

Let $\left(x_{j}\right)_{j=1}^{N}$ be a $\theta \nu$-net for the unit ball $B_{E}$ of $(E,\|\cdot\|)$. We can assume that $N \leq(3 / \theta \nu)^{m}$. Let $F_{0}$ be some fixed $m$-dimensional subspace of $E^{\perp}$. Let $\left(y_{j}\right)_{j=1}^{M}$ be a $\theta \nu$-net for the unit sphere of $\left(F_{0},|\cdot|\right)$. Then we can also assume $M \leq(3 / \theta \nu)^{m}$. Let $s=\left[\theta^{-1} \nu^{-1}\right]+1$. For $1 \leq j \leq N$ and $1 \leq k \leq s$ we define

$$
f_{j k}(z)=\left\|k s^{-1} z+x_{j}\right\| \text {. }
$$


Let $\mu$ denote normalized surface measure on $\left\{z \in E^{\perp}:|z|=1\right\}$. Since each $f_{j k}$ has Lipschitz constant at most $\theta$ (use Proposition 3.3 if $\theta<1$ ) we have (see $[15$, p. 6])

$$
\mu\left\{\left|f_{j k}-a_{j k}\right|>\theta \nu\right\} \leq \sqrt{\pi / 2} e^{-\nu^{2}(n-m-2) / 2}
$$

where $a_{j k}$ is a median value for $f_{j k}$. Let $\lambda$ denote normalized Haar measure on the orthogonal group on $E^{\perp}$. Then

$$
\begin{aligned}
\lambda\left\{U:\left|f_{j k}\left(U y_{l}\right)-a_{j k}\right|>\theta \nu\right\} \leq \sqrt{\pi / 2} e^{-\nu^{2}(n-m-2) / 2}, & \\
& 1 \leq j \leq N, 1 \leq k \leq s, 1 \leq l \leq M .
\end{aligned}
$$

Now, since $m / n \leq \theta^{2} \leq 1$ (by (4.1)),

$$
\begin{aligned}
& M N s \sqrt{\pi / 2} e^{-\nu^{2}(n-m-2) / 2} \\
& \leq\left(\frac{3}{\nu \theta}\right)^{2 m+1} e^{-\nu^{2}(n-m-2) / 2} \leq \theta^{-2 m-1}\left(\frac{n}{m}\right)^{m+1 / 2} e^{-9 m(n-m-2) \log (n / m) / 2 n} \\
& \leq \theta^{-2 m-1}\left(\frac{n}{m}\right)^{m+1 / 2-9 m / 4}=\theta^{-2 m-1}\left(\frac{m}{n}\right)^{5 m / 4-1 / 2} \leq\left(\frac{m}{n}\right)^{m / 4-1}<1 .
\end{aligned}
$$

It follows that there exists $U$ so that

$$
\left|f_{j k}\left(U y_{l}\right)-a_{j k}\right| \leq \theta \nu, \quad 1 \leq j \leq N, 1 \leq k \leq s, 1 \leq l \leq M .
$$

Let $F=U\left(F_{0}\right)$. Suppose $v_{1}, v_{2} \in F$ with $\left|v_{1}\right|=\left|v_{2}\right| \leq 1$. Pick $1 \leq k \leq s$ so that $(k-1) \leq s\left|v_{1}\right| \leq k$. Then we can find $1 \leq l_{1}, l_{2} \leq M$ so that

$$
\left\|k s^{-1} y_{l_{i}}-v_{i}\right\| \leq\left|k s^{-1} y_{l_{i}}-v_{i}\right| \leq 2 \theta \nu, \quad i=1,2 .
$$

Hence

$$
||\left|x_{j}+v_{i} \|-a_{j k}\right| \leq 3 \theta \nu, \quad 1 \leq j \leq N .
$$

This implies that if $x \in B_{E}$ there exists $j$ so that

$$
\left|\left\|x+v_{i}\right\|-a_{j k}\right| \leq 4 \theta \nu, \quad i=1,2 .
$$

Therefore

$$
\left|\left\|x+v_{1}\right\|-\left\|x+v_{2}\right\|\right| \leq 8 \theta \nu .
$$

Now suppose $x \in E$ and $y \in F$. We have

$$
|\|x+y\|-\|x-y\|| \leq 8 \theta \nu|y| \leq 8 d_{X} \theta \nu\|y\| .
$$

If $\|x-y\| \leq\|x+y\|$, this implies that

$$
\|x-y\| \geq\left(1-8 \theta \nu d_{X}\right)\|x+y\| \geq \frac{1}{2}\|x+y\| .
$$

Hence for any $x \in E$ and $y \in F$ we have

$$
\|x-y\| \leq 2\|x+y\|
$$


and thus $E \cap F=\{0\}$. Furthermore,

$$
|y| \leq d_{X}\|y\| \leq \frac{d_{X}}{2}(\|x+y\|+\|x-y\|) \leq \frac{3 d_{X}}{2}\|x+y\| .
$$

If $U$ is orthogonal on $F$ then

$$
\|\widetilde{U}(x+y)\| \leq\|x+y\|+8 \theta \nu|y| \leq\left(1+12 d_{X} \theta \nu\right)\|x+y\| .
$$

This completes the proof for real scalars.

The case of complex scalars is very similar except that one must replace $m, n$ by $2 m, 2 n$ and the orthogonal group by the unitary group.

If $E$ is a normed space, we shall say that a space $Y$ is a Hilbert enlargement of $E$ if $E \subset Y$ and $Y$ can be written as a direct sum $Y=E \oplus H$ where $H$ is isometric to a Hilbert space and

$$
\|e+h\|=\|e+U h\|, \quad e \in E, h \in H,
$$

for every isometry $U$ on $H$. Note that the natural projection $P: Y \rightarrow E$ satisfies $\|I-2 P\|=1$.

Proposition 4.2. Let $X$ be an infinite-dimensional Banach space. If $E$ is an $m$-dimensional subspace of $X$, there is a $2 m$-dimensional Hilbert enlargement of $Y$ which is finitely representable in $X$.

This is a trivial direction from Theorem 4.1.

Proposition 4.3. Let $X$ be an $n$-dimensional normed space. Suppose that $m \in \mathbb{N}$ satisfies $4<m \leq \min \left(\frac{1}{2} n-2,25\left(d_{X}-1\right) n\right)$ and

$$
48 d_{X} \sqrt{\frac{m}{n} \log \frac{n}{m}}<1 .
$$

Let $E$ be an $m$-dimensional subspace of $X$. Then there is a Hilbert enlargement $Y$ of $E$ of dimension $2 m$ and a subspace $Y_{0}$ of $X$ with

$$
d\left(Y, Y_{0}\right) \leq \begin{cases}1+40 d_{X} \sqrt{\frac{m}{n} \log \frac{n}{m}}, & d_{X}>1.04 \\ 1+200 \sqrt{d_{X}-1} \sqrt{\frac{m}{n} \log \frac{n}{m}}, & d_{X} \leq 1.04 .\end{cases}
$$

Proof. Taking $F$ as described in Proposition 4.1 we let $Y_{0}=E+F$ and then define $Y$ to be the space $Y_{0}$ normed by

$$
\|e+f\|_{Y}=\sup _{U \in \mathcal{G}}\|e+U f\|
$$

where $\mathcal{G}$ is the isometry group of $(F,|\cdot|)$. It is then trivial to verify that $Y$ is a Hilbert enlargement of $E$ and the estimates for $d\left(Y, Y_{0}\right)$ hold. 
5. Hilbert enlargements of a one-dimensional space. Let us say that a norm $N$ on $\mathbb{R}^{2}$ is a normalized absolute norm if $(1,0),(0,1)$ is a normalized 1-unconditional basis of $\left(\mathbb{R}^{2}, N\right)$.

We now consider spaces $X$ which are Hilbert enlargements of a onedimensional space. This implies that $X=\mathbb{R} \oplus H$ with a norm of the form

$$
\|(\xi, x)\|=N(|\xi|,\|x\|), \quad \xi \in \mathbb{R}, x \in H,
$$

where $N$ is a normalized absolute norm on $\mathbb{R}^{2}$. We then write $X=\mathbb{R} \oplus_{N} H$.

Our main result in this section is

TheOREM 5.1. There is an absolute constant $C$ so that if $X=\mathbb{R} \oplus_{N} H$ where $N$ is a normalized absolute norm on $\mathbb{R}^{2}, H$ is a real Hilbert space of dimension at least 2 and $\lambda_{X}=1+\varepsilon$ where $0<\varepsilon<1$ then $N$ is a $C \sqrt{\varepsilon}$-perturbation of the standard Euclidean norm on $\mathbb{R}^{2}$.

REMARK. The assumption that $\operatorname{dim} H \geq 2$ is, of course, necessary, since $\lambda_{X}=1$ whenever $\operatorname{dim} X=2$.

In order to prove this theorem it suffices to consider the case when $\operatorname{dim} H=2$ and so we consider $\mathbb{R}^{3}$ with the norm

$$
\left\|\left(\xi_{1}, \xi_{2}, \xi_{3}\right)\right\|=N\left(\xi_{1},\left(\xi_{2}^{2}+\xi_{3}^{2}\right)^{1 / 2}\right) .
$$

We denote the standard Euclidean norm on $\mathbb{R}^{3}$ by $|\cdot|$ and the dual norm by

$$
\left\|\left(\xi_{1}, \xi_{2}, \xi_{3}\right)\right\|_{*}=N^{*}\left(\xi_{1},\left(\xi_{2}^{2}+\xi_{3}^{2}\right)^{1 / 2}\right)
$$

where $N^{*}$ is the normalized absolute norm on $\mathbb{R}^{2}$ dual to $N$.

For $\theta \in \mathbb{R}$ we define

$$
u_{\theta}=(\cos \theta, \sin \theta, 0), \quad v_{\theta}=(-\sin \theta, \cos \theta, 0) .
$$

We also define the operators (writing $\xi=\left(\xi_{1}, \xi_{2}, \xi_{3}\right)$ )

$$
\begin{aligned}
P_{\theta} \xi & =\left(\xi, u_{\theta}\right) u_{\theta}, \quad E_{j} \xi=\xi_{j} e_{j}, \quad j=1,2,3, \\
Q_{\theta} \xi & =\left(\xi, v_{\theta}\right) u_{\theta}, \quad E_{23}=E_{2}+E_{3}
\end{aligned}
$$

and finally the map

$$
J_{\theta}(\xi)=\left(\xi_{1}, \xi_{2} \cos \theta, \xi_{2} \sin \theta\right) .
$$

We denote the adjoint of an operator $T$ by $T^{*}$.

Let $X=\left(\mathbb{R}^{3},\|\cdot\|\right)$. The following lemma follows from Proposition 2.4.

Lemma 5.2. Suppose $\lambda_{X}<1+\varepsilon$. Then there is a continuous map $h=$ $h_{X}:[0, \pi / 2] \rightarrow \mathbb{R}$ with $h(0)=h(\pi / 2)=0$ so that $\left\|P_{\theta}+h(\theta) Q_{\theta}+E_{3}\right\|<1+\varepsilon$.

Proof. We use Proposition 2.4. We argue that there is a continuous map $\Phi:[0, \pi / 2] \rightarrow \mathcal{L}(X)$ so that $\Phi(\theta)$ is a projection onto the two-dimensional subspace $\left\{x:\left(x, v_{\theta}\right)=0\right\}$ and $\|\Phi(\theta)\|<1+\varepsilon$. Let

$$
\Psi(\theta)=\frac{1}{2}\left(I-2 E_{3}\right) \Phi(\theta)\left(I-2 E_{3}\right)+\frac{1}{2} \Phi(\theta)
$$


and then $\Psi$ is also a continuous map satisfying the same conditions as $\Phi$. It is easy to see that $\Psi(\theta)=P_{\theta}+h_{0}(\theta) Q_{\theta}+E_{3}$ where $h_{0}$ is continuous. Since $\left\|P_{0}+E_{3}\right\|=\left\|P_{\pi / 2}+E_{3}\right\|=1$ we may make a small perturbation of $h_{0}$ in a neighborhood of 0 and of $\pi / 2$ to give $h$ with $h(0)=h(\pi / 2)=0$.

Lemma 5.3. There exists $\varepsilon_{0}$ so that if $0<\varepsilon<\varepsilon_{0}$ and $\lambda_{X}<1+\varepsilon$ then $d_{X} \leq 2$ and $\left|h_{X}(\theta)\right| \leq 1$ for $0 \leq \theta \leq \pi / 2$.

Proof. This is a simple compactness argument. If the lemma is false we can find a sequence of normalized absolute norms $N_{k}$ on $\mathbb{R}^{2}$ so that for the associated spaces $X_{k}$, we have $\lambda_{X_{k}} \rightarrow 1$ but either $d_{X_{k}}>2$ or for $h_{k}=h_{X_{k}}$ we have $\max _{0 \leq \theta \leq \pi / 2} h_{k}(\theta)>1$. Then we may pass to a subsequence so that $\left(N_{k}\right)_{k=1}^{\infty}$ converges uniformly on compacta to a limit $N$. It is clear that if $X=\left(\mathbb{R}^{3}, N\right)$ then $\lambda_{X}=1$ and so by Kakutani's theorem $X$ is isometric to a Hilbert space and thus $N$ is the standard Euclidean norm on $\mathbb{R}^{2}$. This shows that $\lim _{k \rightarrow \infty} d_{X_{k}}=1$ and so we must have the second alternative. By passing to a further subsequence we can assume the existence of $0 \leq \theta_{k} \leq \pi / 2$ so that $\left|h_{k}\left(\theta_{k}\right)\right| \geq 1$ and such that $\left(\theta_{k}\right)_{k=1}^{\infty}$ and $\left(h_{k}\left(\theta_{k}\right)\right)_{k=1}^{\infty}$ converge to limits $\theta$ and $\alpha$ respectively. Then $|\alpha| \geq 1$ and $P_{\theta}+\alpha Q_{\theta}$ has norm one on $\left(\mathbb{R}^{3},|\cdot|\right)$, which gives a contradiction.

Let $F(\theta)=\left\|u_{\theta}\right\|$ for $0 \leq \theta \leq \pi / 2$. Our aim will be to show that if $\lambda_{X}<1+\varepsilon$ we have estimates of the type $|h(\theta)| \leq C \sqrt{\varepsilon}$ and $|F(\theta)-1| \leq C \sqrt{\varepsilon}$. In Lemma 5.4 we show that $h$ behaves somewhat like a derivative of $F$. In Lemma 5.5 we prove a very technical estimate on $h(\theta)$ given the value of $h(\phi)$ where $\theta, \phi$ are far enough apart (in fact $|\theta-\phi| \geq \pi / 6$ ). We put these Lemmas together in Lemma 5.4 which gives the promised estimates.

Lemma 5.4. Suppose $0<\varepsilon<\varepsilon_{0}$ and that $\lambda_{X}=1+\varepsilon$.

(i) Suppose $0 \leq \theta \leq \phi \leq \theta+\sqrt{\varepsilon}$. Then there exists $\theta \leq \psi \leq \phi$ so that

$$
|\log F(\phi)-\log F(\theta)-h(\psi)(\phi-\theta)| \leq 4 \varepsilon .
$$

(ii) Suppose $0 \leq \theta \leq \theta+\sqrt{\varepsilon} \leq \phi$. Then there exists $\theta \leq \psi \leq \phi$ so that

$$
\left|h(\psi)-\frac{\log F(\phi)-\log F(\theta)}{\phi-\theta}\right| \leq 8 \sqrt{\varepsilon} .
$$

Proof. Assume $0<\theta, \phi<\pi / 2$. We observe that

$$
F(\phi)\left\|u_{\theta}+h(\theta) v_{\theta}\right\|_{*} \geq\left(u_{\phi}, u_{\theta}+h(\theta) v_{\theta}\right)=\cos (\phi-\theta)+h(\theta) \sin (\phi-\theta) .
$$

Now consider the rank one operator $P_{\theta}+h(\theta) Q_{\theta}$; then

$$
\left\|P_{\theta}+h(\theta) Q_{\theta}\right\|=\left\|\left(I-E_{3}\right)\left(P_{\theta}+h(\theta) Q_{\theta}+E_{3}\right)\right\|<1+\varepsilon
$$

so that

$$
\left\|u_{\theta}+h(\theta) v_{\theta}\right\|_{*} F(\theta)<1+\varepsilon .
$$


Hence

$$
(1+\varepsilon) \frac{F(\phi)}{F(\theta)} \geq \cos (\phi-\theta)+h(\theta) \sin (\phi-\theta) .
$$

Thus

$\log F(\phi)-\log F(\theta) \geq-\log (1+\varepsilon)+\log \left(1-2 \sin ^{2}\left(\frac{1}{2}(\phi-\theta)\right)+h(\theta) \sin (\phi-\theta)\right)$.

Now assume $|\theta-\phi| \leq \sqrt{\varepsilon}$. We use the simple estimates $|\sin t| \leq|t|$ and $|\sin t-t| \leq \frac{1}{2} t^{2}$. Then, since $\varepsilon<\varepsilon_{0}$ and consequently $|h(\theta)| \leq 1$,

$$
|h(\theta) \sin (\phi-\theta)-h(\theta)(\phi-\theta)| \leq \frac{1}{2} \varepsilon \text {. }
$$

Thus we have

$$
\begin{aligned}
\log F(\phi)-\log F(\theta) & \geq-\varepsilon+\log (1+h(\theta)(\phi-\theta)-\varepsilon) \\
& \geq h(\theta)(\phi-\theta)-2 \varepsilon-\frac{1}{2}(|\phi-\theta|+\varepsilon)^{2} \geq h(\theta)(\phi-\theta)-4 \varepsilon .
\end{aligned}
$$

Similarly,

$$
\log F(\theta)-\log F(\phi) \geq h(\phi)(\theta-\phi)-4 \varepsilon,
$$

so that if we also assume $\theta<\phi$ then

$$
h(\theta)(\phi-\theta)-4 \varepsilon \leq \log F(\phi) / F(\theta) \leq h(\phi)(\phi-\theta)+4 \varepsilon
$$

whenever $0<\theta<\phi \leq \theta+\sqrt{\varepsilon}$. Since $h$ is continuous, the intermediate value theorem implies (i).

If $\phi \geq \theta+\sqrt{\varepsilon}$, we select $n$ to be the smallest integer such that $(\phi-\theta) / n$ $\leq \sqrt{\varepsilon}$. Then if $\theta_{k}=\theta+\frac{k}{n}(\phi-\theta)$ for $1 \leq k \leq n$, we have

$$
\frac{1}{n} \sum_{k=0}^{n-1} h\left(\theta_{k}\right) \leq \frac{\log F(\phi)-\log F(\theta)+4 n \varepsilon}{\phi-\theta},
$$

which implies, since $\frac{1}{2} n \leq(n-1) \leq(\phi-\theta) \varepsilon^{-1 / 2}$,

$$
\frac{1}{n} \sum_{k=0}^{n-1} h\left(\theta_{k}\right) \leq \frac{\log F(\phi)-\log F(\theta)}{\phi-\theta}+8 \sqrt{\varepsilon} .
$$

Hence there exists $k$ with $0 \leq k \leq n-1$ and

Similarly,

$$
h\left(\theta_{k}\right) \leq \frac{\log F(\phi)-\log F(\theta)}{\phi-\theta}+8 \sqrt{\varepsilon} .
$$

$$
\frac{1}{n} \sum_{k=1}^{n} h\left(\theta_{k}\right) \geq \frac{\log F(\phi)-\log F(\theta)}{\phi-\theta}-8 \sqrt{\varepsilon}
$$

so there exists $j$ with $1 \leq j \leq n$ and

$$
h\left(\theta_{j}\right) \geq \frac{\log F(\phi)-\log F(\theta)}{\phi-\theta}-8 \sqrt{\varepsilon} .
$$

(ii) again follows by the intermediate value theorem since $h$ is continuous. 
LEMMA 5.5. There is an absolute constant $C_{0}$ so that if $\lambda_{X}<1+\varepsilon$ where $0<\varepsilon<\varepsilon_{0}$, then

$$
|h(\theta)| \leq C_{0} \frac{|h(\phi)|+\sqrt{\varepsilon}}{\sin (2 \phi)}, \quad 0 \leq \theta, \phi \leq \pi / 2,|\theta-\phi| \geq \pi / 6 .
$$

Proof. Let us suppose $0 \leq \theta \leq \theta+\pi / 6 \leq \phi \leq \pi / 2$. We will show that

$$
\begin{aligned}
& |h(\theta)| \leq(4|h(\phi)|+9 \sqrt{\varepsilon}) \sec \phi, \\
& |h(\phi)| \leq(9|h(\theta)|+40 \sqrt{\varepsilon}) \csc \theta .
\end{aligned}
$$

Clearly, we may combine (5.2) and (5.3) into the single statement (5.1).

We will define $\psi$ with $0 \leq \psi \leq \pi / 2$ via the formula

$$
\cos \psi=\frac{\tan \theta}{\tan \phi} \text {. }
$$

We observe that

$$
\cos \psi \leq \max _{0 \leq \theta \leq \pi / 3} \frac{\tan \theta}{\tan (\theta+\pi / 6)}=\frac{\tan (\pi / 6)}{\tan (\pi / 3)}=1 / 3 .
$$

Hence

$$
\sin \psi \geq 2 \sqrt{2} / 3>9 / 10
$$

Set $x=J_{\psi} u_{\phi}$ and $y=J_{\psi} v_{\phi}$. We also define $f=(0,-\sin \psi, \cos \psi)$, so that $f$ is a unit vector in $H$ orthogonal to $E_{23} x$ and $E_{23} y$.

From the form of $\|\cdot\|$ and its dual it is clear that

$$
\|x\|=\left\|u_{\phi}\right\| \quad \text { and } \quad\|x+h(\phi) y\|_{*}=\left\|u_{\phi}+h(\phi) v_{\phi}\right\|_{*} .
$$

Hence

$$
\|x\|\|x+h(\phi) y\|_{*} \leq\left\|P_{\phi}+h(\phi) Q_{\phi}\right\|<1+\varepsilon .
$$

Let us define

$$
x^{\prime}=\left(P_{\theta}+h(\theta) Q_{\theta}^{*}+E_{3}\right) x, \quad y^{\prime}=\left(P_{\theta}+h(\theta) Q_{\theta}^{*}+E_{3}\right) y .
$$

Then

$$
\left\|x^{\prime}+h(\phi) y^{\prime}\right\|_{*} \leq(1+\varepsilon)\|x+h(\phi) y\|_{*}
$$

and

$$
\left(x, x^{\prime}\right)=(x, x)=1, \quad\left(x, y^{\prime}\right)=(x, y)=0,
$$

since $x$ is in the range of the projection $P_{\theta}+h(\theta) Q_{\theta}+E_{3}$.

Because of the special form of the norm it is clear that we must have

$$
\begin{aligned}
& \left(E_{1} x, E_{1}\left(x^{\prime}+h(\phi) y^{\prime}\right)\right)+\left|E_{23} x\right|\left|E_{23}\left(x^{\prime}+h(\phi) y^{\prime}\right)\right| \\
& \quad \leq\|x\|\left\|x^{\prime}+h(\phi) y^{\prime}\right\|_{*} \leq(1+\varepsilon)\|x\|\|x+h(\phi) y\|_{*} \leq(1+\varepsilon)^{2}<1+3 \varepsilon .
\end{aligned}
$$

Hence

$$
\left|E_{23} x\right|\left|E_{23}\left(x^{\prime}+h(\phi) y^{\prime}\right)\right|-\left(E_{23} x, E_{23}\left(x^{\prime}+h(\phi) y^{\prime}\right)\right) \leq 3 \varepsilon .
$$


Now $\left|E_{23} x\right| \leq 1$ and $\left|E_{23}\left(x^{\prime}+h(\phi) y^{\prime}\right)\right| \leq 1+3 \varepsilon<2$ so we also have

$$
\left|E_{23} x\right|\left|E_{23}\left(x^{\prime}+h(\phi) y^{\prime}\right)\right|+\left(E_{23} x, E_{23}\left(x^{\prime}+h(\phi) y^{\prime}\right)\right)<4
$$

and consequently

$$
\left|E_{23} x\right|^{2}\left|E_{23}\left(x^{\prime}+h(\phi) y^{\prime}\right)\right|^{2}-\left(E_{23} x, E_{23}\left(x^{\prime}+h(\phi) y^{\prime}\right)\right)^{2} \leq 12 \varepsilon .
$$

Recalling our choice of $f$ we may write, for suitable $\alpha$ and $\beta$,

$$
E_{23}\left(x^{\prime}+h(\phi) y^{\prime}\right)=\alpha E_{23} x+\beta f
$$

and then we have

$$
\left|E_{23} x\right|^{2}\left(\alpha^{2}\left|E_{23} x\right|^{2}+\beta^{2}\right)-\alpha^{2}\left|E_{23} x\right|^{4} \leq 12 \varepsilon
$$

or

$$
\beta^{2}\left|E_{23} x\right|^{2} \leq 12 \varepsilon
$$

Thus, the above inequality gives

$$
\left|E_{23} x\right|^{2}\left(x^{\prime}-x+h(\phi) y^{\prime}, f\right)^{2} \leq 12 \varepsilon .
$$

Since $\left|E_{23} x\right|=\sin \phi \geq 1 / 2$ we conclude that

$$
\left|\left(f, x^{\prime}-x+h(\phi) y^{\prime}\right)\right| \leq 8 \sqrt{\varepsilon} .
$$

Now

$$
\begin{aligned}
x^{\prime}-x & =h(\theta) Q_{\theta}^{*} x=h(\theta)\left(x, u_{\theta}\right) v_{\theta}, \\
y^{\prime} & =\left(P_{\theta}+h(\theta) Q_{\theta}^{*}+E_{3}\right) y=\left(y, u_{\theta}\right) u_{\theta}+h(\theta)\left(y, u_{\theta}\right) v_{\theta}+\left(y, e_{3}\right) e_{3} .
\end{aligned}
$$

Since $\cos \psi=\tan \theta \cot \phi$ we have

$$
\left(x, u_{\theta}\right)=\cos \phi \cos \theta+\sin \phi \sin \theta \cos \psi=\frac{\cos \phi}{\cos \theta}
$$

and

$$
\begin{aligned}
\left(y, u_{\theta}\right) & =\cos \phi \sin \theta \cos \psi-\sin \phi \cos \theta=\frac{\cos ^{2} \phi \sin ^{2} \theta-\sin ^{2} \phi \cos ^{2} \theta}{\sin \phi \cos \theta} \\
& =-\frac{\sin (\phi-\theta) \sin (\phi+\theta)}{\sin \phi \cos \theta} .
\end{aligned}
$$

Also

$$
\begin{aligned}
\left(y, e_{3}\right) & =\cos \phi \sin \psi, & & \left(f, v_{\theta}\right)=-\sin \psi \cos \theta \\
\left(f, u_{\theta}\right) & =-\sin \psi \sin \theta, & & \left(f, e_{3}\right)=\cos \psi
\end{aligned}
$$

Thus

$$
\left(f, x^{\prime}-x+h(\phi) y^{\prime}\right)=-a h(\theta)+b h(\phi)+c h(\theta) h(\phi)
$$


where

$$
\begin{aligned}
a & =a(\theta, \phi)=-\left(u_{\theta}, x\right)\left(f, v_{\theta}\right)=\sin \psi \cos \phi, \\
b & =b(\theta, \phi)=\left(u_{\theta}, y\right)\left(f, u_{\theta}\right)+\left(e_{3}, y\right)\left(f, e_{3}\right) \\
& =\sin \psi \sin \theta \frac{\sin (\phi-\theta) \sin (\phi+\theta)}{\sin \phi \cos \theta}+\cos \phi \sin \psi \cos \psi, \\
c & =c(\theta, \phi)=\left(u_{\theta}, y\right)\left(f, v_{\theta}\right) .
\end{aligned}
$$

From this we get the following estimates since $\phi-\theta \geq \pi / 6$ and $\phi+\theta \leq 5 \pi / 6$ :

$$
\frac{9}{10} \cos \phi \leq a \leq 1, \quad \frac{9}{40} \sin \theta \leq b \leq 2, \quad 0 \leq c \leq 1
$$

Now combining (5.4) and (5.5) we have

$$
|a(\theta, \phi)||h(\theta)| \leq 8 \sqrt{\varepsilon}+3|h(\phi)|,
$$

and so we obtain (5.2). Similarly,

$$
|b(\theta, \phi)||h(\phi)| \leq 8 \sqrt{\varepsilon}+2|h(\theta)|,
$$

and hence we deduce (5.3).

Lemma 5.6. Suppose $\lambda_{X}<1+\varepsilon$ where $0<\varepsilon<1$. Then there is an absolute constant $C$ so that

$$
1-C \sqrt{\varepsilon} \leq F(\theta) \leq 1+C \sqrt{\varepsilon} \quad \text { and } \quad|h(\theta)| \leq C \sqrt{\varepsilon} \quad \text { for } 0 \leq \theta \leq \pi / 2 .
$$

Proof. It suffices to produce a constant which works under the additional assumption that $0<\varepsilon<\min \left(1 / 64, \varepsilon_{0}\right)$. Let

$$
M_{0}=\max _{0 \leq \theta \leq \pi / 2}|h(\theta)|, \quad M_{1}=\max _{0 \leq \theta \leq \pi / 2}|\log F(\theta)| .
$$

Assume $0 \leq \phi \leq \pi / 4$. Then inequality (5.1) of Lemma 5.5 gives

$$
|h(5 \pi / 12)| \leq C_{0} \frac{|h(\phi)|+\sqrt{\varepsilon}}{\sin (2 \phi)}
$$

and then another use of (5.1) shows that

$$
|h(\theta)| \leq 2 C_{0}^{2} \frac{|h(\phi)|+\sqrt{\varepsilon}}{\sin (2 \phi)}+2 C_{0} \sqrt{\varepsilon}, \quad 0 \leq \theta \leq \pi / 4 .
$$

In particular, we get an estimate when $\theta=\pi / 12$ and the same reasoning gives

$$
|h(\theta)| \leq 4 C_{0}^{3} \frac{|h(\phi)|+\sqrt{\varepsilon}}{\sin (2 \phi)}+\left(4 C_{0}^{2}+2 C_{0}\right) \sqrt{\varepsilon}, \quad \pi / 4 \leq \theta \leq \pi / 2 .
$$

Similar arguments apply when $\pi / 4 \leq \phi \leq \pi / 2$. We can combine all these statements into the following inequality:

$$
M_{0} \leq C_{1} \frac{|h(\phi)|+\sqrt{\varepsilon}}{\sin (2 \phi)}, \quad 0<\phi<\pi / 2
$$

where $C_{1}$ is an absolute constant. 
Using Lemma 5.4, since $\log F(0)=\log F(\pi / 2)=0$, we obtain an estimate $|\log F(\theta)| \leq\left(M_{0}+8 \sqrt{\varepsilon}\right) \min (|\theta|,|\pi / 2-\theta|), \quad \sqrt{\varepsilon} \leq \theta \leq \pi / 2-\sqrt{\varepsilon}$, and it follows that (for an absolute constant $C_{2}$ )

$$
|\log F(\theta)| \leq C_{2}\left(M_{0}+8 \sqrt{\varepsilon}\right) \sin (2 \theta), \quad \sqrt{\varepsilon} \leq \theta \leq \pi / 2-\sqrt{\varepsilon} .
$$

We now turn to estimating $M_{1}$. Fix $0 \leq \theta \leq \pi / 2$ so that $|\log F(\theta)|=M_{1}$. If $\theta \leq 2 \sqrt{\varepsilon}$ then $\left|u_{\theta}-e_{1}\right| \leq 2 \sqrt{\varepsilon}$ so that $\left\|u_{\theta}-e_{1}\right\| \leq 4 \sqrt{\varepsilon}$ and hence $1-4 \sqrt{\varepsilon} \leq e^{M_{1}} \leq 1+4 \sqrt{\varepsilon}$. Since $\varepsilon<1 / 64$ this gives us an estimate $M_{1} \leq 8 \sqrt{\varepsilon}$. If $\theta \geq \pi / 2-2 \sqrt{\varepsilon}$ we obtain a similar estimate.

If $2 \sqrt{\varepsilon} \leq \theta \leq \pi / 2-2 \sqrt{\varepsilon}$ we use Lemma 5.4 to produce a point $\phi$ with $|\phi-\theta| \leq \sqrt{\varepsilon}$ and $|h(\phi)| \leq 4 \sqrt{\varepsilon}$. For example, suppose $F$ assumes its maximum at $\theta$. Then by Lemma 5.4 there exists $\theta-\sqrt{\varepsilon} \leq \psi_{0} \leq \theta$ so that

$$
\sqrt{\varepsilon} h\left(\psi_{0}\right) \geq \log F(\theta)-\log F(\theta-\sqrt{\varepsilon})-4 \varepsilon \geq-4 \varepsilon
$$

and a point $\theta \leq \psi_{1} \leq \theta+\sqrt{\varepsilon}$ so that

$$
\sqrt{\varepsilon} h\left(\psi_{1}\right) \leq 4 \varepsilon .
$$

Since $h$ is continuous the intermediate value theorem gives us $\phi$ with $\psi_{0} \leq$ $\phi \leq \psi_{1}$. The case when $F$ assumes a minimum at $\theta$ is completely analogous.

Now using (5.6) above we have

$$
M_{0} \leq 5 C_{1} \sqrt{\varepsilon} \csc (2 \phi)
$$

and hence by (5.7),

$$
M_{1} \leq C_{2}\left(5 C_{1} \csc (2 \phi)+8\right) \sin (2 \theta) \sqrt{\varepsilon}
$$

or

$$
M_{1} \leq C_{3} \frac{\sin (2 \theta)}{\sin (2 \phi)} \sqrt{\varepsilon}
$$

Now

$$
\frac{\sin (2 \theta)}{\sin (2 \phi)} \leq 1+\cot (2 \phi)|\sin 2(\theta-\phi)| \leq 1+\cot (2 \sqrt{\varepsilon}) \sin (2 \sqrt{\varepsilon}) \leq 2 .
$$

We therefore have an estimate $M_{1} \leq 2 C_{3} \sqrt{\varepsilon}$, which gives the first part of the conclusion of the lemma.

To reach the second statement we observe that $|\log F(\pi / 3)-\log F(\pi / 6)|$ $\leq 2 M_{1}$ and so there exists $\pi / 6 \leq \phi \leq \pi / 3$ with

$$
|h(\phi)| \leq 12 \pi^{-1} M_{1}+8 \sqrt{\varepsilon} \leq C_{4} \sqrt{\varepsilon},
$$

and then applying (5.6) gives an estimate $M_{0} \leq C_{5} \sqrt{\varepsilon}$.

Proof of Theorem 5.1. This follows directly by an appeal to Lemma 3.2. We need only show an estimate for $0<\varepsilon<\varepsilon_{0}$. Take $\nu=2 \sin (\sqrt{\varepsilon} / 2)$. By Lemmas 5.4 and 5.6 we obtain an estimate $|F(\theta)-F(\phi)| \leq C \varepsilon$ if $|\theta-\phi| \leq \sqrt{\varepsilon}$. 
Thus for $x, x^{\prime} \in \mathbb{R}^{2}$,

$$
|x|=\left|x^{\prime}\right|=1,\left|x-x^{\prime}\right| \leq \nu \Rightarrow\left|\|x\|-\left\|x^{\prime}\right\|\right| \leq C \varepsilon .
$$

Lemma 3.2 now gives the theorem.

TheOrem 5.7. There is an absolute constant $C$ so that if $X=\mathbb{C} \oplus_{N} H$ where $N$ is a normalized absolute norm on $\mathbb{R}^{2}, H$ is a complex Hilbert space of dimension at least 2 and $\lambda_{X}=1+\varepsilon$ where $0<\varepsilon<1$ then $N$ is a $C \sqrt{\varepsilon}$-perturbation of the standard Euclidean norm on $\mathbb{R}^{2}$.

Proof. This is now a simple deduction. Let $\left(e_{j}\right)_{j \in \mathbb{A}}$ be an orthonormal basis of $H$ and consider the real-linear subspace $Z$ of $X=\mathbb{C} \oplus_{N} H$ of all $(\xi, h)$ such that $\xi \in \mathbb{R}$ and $\left(h, e_{j}\right) \in \mathbb{R}$ for all $j \in \mathbb{A}$. Then there is a real-linear projection $P: \mathbb{C} \oplus_{N} H$ onto $Z$ with ker $P=i Z$ and $\|P\|=1$. If $E$ is a closed subspace of the real Banach space $Z$ then then there is a complex-linear projection $Q: X \rightarrow E+i E$ with $\|Q\| \leq 1+\varepsilon$. Then $P Q$ is a real-linear projection onto $E$ so that $\lambda_{Z} \leq 1+\varepsilon$ and the result follows from Theorem 5.1.

\section{The main theorem}

Proposition 6.1. There is a universal constant $C$ so that if $X$ is a finite-dimensional Banach space and $Y$ is a Hilbert enlargement of $X$ with $\operatorname{dim} Y=2 \operatorname{dim} X$ such that $\lambda_{Y}<2$ then

$$
d_{X}-1 \leq C \sqrt{\lambda_{Y}-1} .
$$

Proof for real scalars. Suppose $\lambda_{Y}=1+\varepsilon$, and let $m=\operatorname{dim} X$. Let $Y=X \oplus H$ where $H$ is an $m$-dimensional Hilbert space.

If $u \in X$ with $\|u\|=1$ then the span $F_{u}$ of $u$ and $H$ has a norm of the form

$$
\|\xi u+h\|=N_{u}(|\xi|,\|h\|)
$$

where $N_{u}$ is a normalized absolute norm on $\mathbb{R}^{2}$. By Theorem 5.1, since $\lambda_{F_{u}} \leq$ $1+\varepsilon$ we deduce that $N_{x}$ is a $\delta$-perturbation of the Euclidean norm on $\mathbb{R}^{2}$ where $\delta \leq C \sqrt{\varepsilon}$ for some absolute constant $C$. In particular, if $\varepsilon$ is small enough, say $0<\varepsilon<\varepsilon_{0}$, we have $\delta<1 / 3$.

It follows that on $F_{u}$ the norm $\|\cdot\|$ is a $\delta$-perturbation of the norm $|\cdot|_{u}$ given by the inner product

$$
\left(\xi_{1} x+h_{1}, \xi_{2} x+h_{2}\right)_{u}=\xi_{1} \xi_{2}+\left(h_{1}, h_{2}\right) .
$$

In particular, since $\|u\|=1=|u|_{u}$ and $\|h\|=|h|_{u}$ for $h \in H$ it follows that

$$
\|\xi u+h\| \geq(1-\delta)\left(|\xi|^{2}+\|h\|^{2}\right)^{1 / 2} .
$$

This means that for $x \in X$ and $h \in H$ we have

$$
\|x+h\| \geq(1-\delta)\left(\|x\|^{2}+\|h\|^{2}\right)^{1 / 2} .
$$


We let $S: X \rightarrow H$ be an operator with $d_{X}^{-1}\|x\| \leq\|S x\| \leq\|x\|$ for $x \in X$. By Theorem 2.1, $d_{X} \leq M$ for some absolute constant $M$. Proceeding as in [13] and [10] we consider the subspace $G$ of $Y$ given by $G=\{x+S x: x \in X\}$. Then there is a projection $P: Y \rightarrow G$ with $\|P\| \leq 1+\varepsilon$. Let $G^{\prime}=\operatorname{ker} P$. Suppose $y+h \in G^{\prime}$ where $y \in X$ and $h \in H$ with $\|h\|=1$. Then $h=S x$ where $1 \leq\|x\| \leq d_{X}$. Note that $x+h=P(x+h)=P(x-y)$. Hence

Thus

$$
\|x+h\| \leq(1+\varepsilon)\|x-y\| .
$$

and so

$$
(1-\delta)^{2}\left(\|x\|^{2}+1\right) \leq(1+\varepsilon)^{2}\|x-y\|^{2}
$$

$$
\frac{1-C \sqrt{\varepsilon}}{1+\varepsilon} \leq \frac{\|x\|+\|y\|}{\sqrt{\|x\|^{2}+1}} \leq \frac{M}{\sqrt{M^{2}+1}}+\frac{\|y\|}{\sqrt{2}} .
$$

It follows that for some $\varepsilon_{1}>0$, if $0<\varepsilon<\varepsilon_{1}$ we have an estimate $\|y\| \geq c>0$, for some universal choice of $c>0$. Thus, in particular, $G^{\prime} \cap H=\{0\}$ and so $G^{\prime}$ can be expressed in the form $G^{\prime}=\{x-T x: x \in X\}$ where $T: X \rightarrow H$ is some linear map. Furthermore, $T$ satisfies a bound $\|T\| \leq M^{\prime}$ (where $M^{\prime}=1 / c$ is independent of $\varepsilon$ for $\left.0<\varepsilon<\varepsilon_{1}\right)$.

Now fix $u \in X$ with $\|u\|=1$. Let $x=(u+S u) /\|u+S u\|$ and $y=$ $(u-T u) /\|u-T u\|$. Then

$$
\|x\| \leq(1+\varepsilon)\|x+t y\|, \quad-\infty<t<\infty .
$$

Note that $x, y \in F_{u}$ and recall that $\delta<1 / 3$, so that we can apply Lemma 3.5 to deduce that if $\varepsilon<\varepsilon_{1}$ then

$$
\left|(x, y)_{u}\right| \leq \delta+\sqrt{2 \varepsilon}
$$

Hence

$$
\begin{aligned}
|1-(S u, T u)| & \leq(\delta+\sqrt{2 \varepsilon})\|u+S u\|\|u-T u\| \\
& \leq(\delta+\sqrt{2 \varepsilon})\left(M^{\prime}+1\right)(M+1) \leq C_{1} \sqrt{\varepsilon}
\end{aligned}
$$

where $C_{1}$ is some constant, independent of $\varepsilon$.

It follows that for $x \in X$ we have

$$
\left|\|x\|^{2}-(S x, T x)\right| \leq C_{1} \sqrt{\varepsilon}\|x\|^{2} .
$$

Thus, for $\varepsilon$ small enough so that $C_{1} \sqrt{\varepsilon}<1 / 2$ we can define a Euclidean norm on $X$ by $|x|_{X}^{2}=(S x, T x)$, and this shows that $d_{X} \leq 1+C_{2} \sqrt{\varepsilon}$ for a suitable constant $C_{2}$.

Proof for complex scalars. This is a very similar argument. We use Theorem 5.7 in place of Theorem 5.1 and define $S, T$ (this time complex-linear) as above. Then treating $Y$ as a real normed space and $H$ as a real Hilbert space with the inner product $\operatorname{Re}(x, y)$ we deduce that

$$
\left|\|x\|^{2}-\operatorname{Re}(S x, T x)\right| \leq C_{1} \sqrt{\varepsilon}\|x\|^{2} .
$$


This is enough to show that $\sqrt{\operatorname{Re}(S x, T x)}$ defines a norm on $E$ which makes it a complex Hilbert space (provided $\varepsilon$ is small enough).

TheOREM 6.2. There is a universal constant $C$ so that if $X$ is a (real or complex) infinite-dimensional Banach space and $\lambda_{X}<2$ then

$$
d_{X}-1 \leq C \sqrt{\lambda_{X}-1}
$$

Proof. This now follows directly from Propositions 4.2 and 6.1 since we get a uniform estimate $d_{E} \leq 1+C \sqrt{d_{X}-1}$ for all finite-dimensional subspaces of $X$.

EXAmple 1. Let us present an example to show that Theorem 6.2 is up to the constant $C$ the best possible. For any $0<\delta<1 / 3$ let us take $X=\mathbb{R} \oplus_{N} H$ where

$$
N(\xi, \eta)=(1-\delta)\left(\xi^{2}+\eta^{2}\right)^{1 / 2}+\delta \max (|\xi|,|\eta|) .
$$

Then $N$ is an $\delta$-perturbation of $(1-\delta)\left(\xi^{2}+\eta^{2}\right)^{1 / 2}$ and so, by Theorem 3.6, $\lambda_{X} \leq 1+\delta^{2}$. On the other hand, it is clear by a symmetry argument that $d_{X}$ can be computed by comparing $N$ to a norm of the type $\alpha\left(\xi^{2}+\eta^{2}\right)^{1 / 2}$ where $1-\delta \leq \alpha \leq 1$. But then

$$
N\left(\frac{1}{\sqrt{2}}, \frac{1}{\sqrt{2}}\right)=1-\delta\left(1-\frac{1}{\sqrt{2}}\right)
$$

so that

$$
\begin{aligned}
\max _{\xi^{2}+\eta^{2}=1} \mid N(\xi, \eta)- & \alpha\left(\xi^{2}+\eta^{2}\right)^{1 / 2} \mid \\
& \geq \max \left(1-\alpha, \delta\left(1-\frac{1}{\sqrt{2}}\right)-(1-\alpha)\right) \geq \frac{\delta}{2-\sqrt{2}} .
\end{aligned}
$$

It follows that $d_{X}-1 \geq c \delta$ for some $c>0$ and $\lambda_{X}-1 \leq 1+\delta^{2}$.

EXAMPLE 2. One cannot expect to prove a converse estimate of the type $\lambda_{X}-1 \leq C\left(d_{X}-1\right)^{2}$. Let us give an example here by taking $X=X_{\delta}=$ $\mathbb{R} \oplus_{N} H$ where

$$
N(\xi, \eta)=\max \left(|\xi|,\left(\left(1-\delta^{2}\right) \xi^{2}+\eta^{2}\right)^{1 / 2}\right)
$$

In this case it is easy to see that $d_{X} \leq 1+c \delta^{2}$ for some constant $c$. On the other hand, $N(1,0)=1$ while $N\left(\left(1-\delta^{2}\right)^{1 / 2}, \delta\right)=\left(1-\delta^{2}+\delta^{4}\right)^{1 / 2}$, so that the Lipschitz constant of $N(\xi, \eta)-\left(\xi^{2}+\eta^{2}\right)^{1 / 2}$ cannot be better than $c_{1} \delta$ where $c_{1}>0$. Now Theorem 5.1 implies that we have an estimate $\lambda_{X} \geq 1+c_{2} \delta^{2}$ for some $c_{2}>0$. Note that this also provides an example to establish the sharpness up to a constant of Proposition 3.3.

It seems reasonable to conjecture that there is a constant $C$ so that if $\lambda_{X}<2$ then $X$ is a $C \sqrt{\lambda_{X}-1}$-perturbation of some Hilbert space. This would provide a nice converse to Theorem 3.6. Of course, for the special class of spaces $X$ so that $\|x\|^{2 n}$ is a polynomial this can be proved using 
Propositions 3.4 and 3.6. In general, it relates to the question whether we can find a Euclidean norm $|\cdot|$ of $X$ so that we have a projection on every subspace with small norm in both the original norm on $X$ and the approximating Euclidean norm.

Theorem 6.3. Suppose $0<\varepsilon<1 / 200$. Suppose $X$ is a Banach space on which there is an equivalent Euclidean norm $|\cdot|$ so that for every $x \in X$ there is a projection $P$ on $[x]$ with $\|P\| \leq 1+\varepsilon$ and $|P| \leq 1+\varepsilon$. Then there is a Euclidean norm $|\cdot|^{\prime}$ on $X$ so that $\|\cdot\|$ is a $25 \sqrt{\varepsilon}$-perturbation of $|\cdot|^{\prime}$.

Proof. We normalize $|\cdot|$ by assuming that there exists $x_{0}$ so that $\left|x_{0}\right|=$ $\left\|x_{0}\right\|=1$. Then we shall take $|\cdot|^{\prime}=|\cdot|$.

Suppose $|x|=1$. Then there exists a rank-one projection $P_{x}: H \rightarrow[x]$ with $\left\|P_{x}\right\|,\left|P_{x}\right| \leq 1+\varepsilon$. Thus $P_{x} v=\left(v, x+y_{x}\right) x$ where $\left(x, y_{x}\right)=0$. Now $\left|P_{x}\right|=1+\left|y_{x}\right|^{2}$ so that $\left|y_{x}\right| \leq \sqrt{\varepsilon}$. Also, $\left\|P_{x}\right\|=\|x\|\left\|x+y_{x}\right\|_{*}<1+\varepsilon$.

Now suppose $|x|=\left|x^{\prime}\right|=1$. Then $\left(x, x-x^{\prime}\right)=1-\left(x, x^{\prime}\right)=\frac{1}{2}\left|x-x^{\prime}\right|^{2}$. Let us suppose $\left|x-x^{\prime}\right| \leq \sqrt{\varepsilon}$. Choosing $y=y_{x}$ as above we have

$$
\left(x-x^{\prime}, x+y\right) \leq \sqrt{\varepsilon}\left|x-x^{\prime}\right|+\frac{1}{2}\left|x-x^{\prime}\right|^{2} \leq \frac{3}{2} \varepsilon .
$$

Thus

$$
\left(x^{\prime}, x+y\right) \geq 1-\frac{3}{2} \varepsilon .
$$

Hence

$$
\left\|x^{\prime}\right\|\|x+y\|_{*} \geq 1-\frac{3}{2} \varepsilon \quad \text { and } \quad \frac{\left\|x^{\prime}\right\|}{\|x\|} \geq(1+\varepsilon)^{-1}\left(1-\frac{3}{2} \varepsilon\right) \geq(1+3 \varepsilon)^{-1},
$$

since $\varepsilon<\frac{1}{12}$. It now follows, by symmetry, that

$$
\left|\log \|x\|-\log \left\|x^{\prime}\right\|\right| \leq 3 \varepsilon .
$$

Hence for any $x$ with $|x|=1$ we have

$$
\left|\log \|x\|-\log \left\|x_{0}\right\|\right| \leq 3\left(\frac{\pi}{2 \sqrt{\varepsilon}}+1\right) \varepsilon \leq 6 \sqrt{\varepsilon} .
$$

Thus

$$
\|x\| \leq e^{6 \sqrt{\varepsilon}} \leq(1-6 \sqrt{\varepsilon})^{-1} \leq 1+12 \sqrt{\varepsilon},
$$

since $\varepsilon<1 / 144$. Similarly,

$$
\|x\| \geq 1-12 \sqrt{\varepsilon} .
$$

If $\left\|x-x^{\prime}\right\| \leq \sqrt{\varepsilon}$ we have

$$
\|x\|-\left\|x^{\prime}\right\| \leq 3 \sqrt{\varepsilon} e^{1+12 \sqrt{\varepsilon}} \leq 6 \sqrt{\varepsilon} .
$$

Hence, by Lemma $3.2,\|\cdot\|$ is a $25 \sqrt{\varepsilon}$-perturbation of $|\cdot|$. 
7. The symmetry problem. We now consider a similar problem for the symmetry constant $\sigma_{X}$. We will also study the reflection constant $\varrho_{X}$ (see Section 2). We also define the transitivity constant $\tau_{X}$ to be the infimum of all constants $\tau$ such that whenever $x, y \in \partial B_{X}$ then there exists an invertible operator $U$ with $U x \in[y]$ and $\|U\|\left\|U^{-1}\right\|<\tau$.

The corresponding isometric constants have been studied in the literature. A Banach space $X$ is called transitive if whenever $x, y \in \partial B_{X}$ there is an invertible isometry $U: X \rightarrow X$ with $U x=y$. It is a classic unsolved problem of Banach and Mazur whether every separable transitive space is isometric to a Hilbert space (this problem is discussed in [18]). A Banach space is called almost transitive if whenever $x, y \in \partial B_{X}$ and $\varepsilon>0$ there is an invertible isometry $U: X \rightarrow X$ with $\|U x-y\|<\varepsilon$. It is clear that every finite-dimensional almost transitive space is isometrically Hilbertian (see [18]), but in infinite dimensions things are more complicated: indeed, the spaces $L_{p}[0,1]$ for $1 \leq p<\infty$ are almost transitive (see [18]). Note that this implies that $\tau_{L_{p}}=1$ for $1 \leq p<\infty$.

Skorik and Zaidenberg [19] showed that a Banach space with one nontrivial isometric reflection is isometric to a Hilbert space; of course, these hypotheses imply that $\varrho_{X}=1$, since if $R$ is a non-trivial reflection and $U$ is invertible then $U^{-1} R U$ is also a reflection. This result was improved by Randrianantoanina [17] by requiring only a norm-one projection onto a hyperplane; earlier a more restricted version of this result was proved by Odinec [16].

The next lemma extends the fact that there is at most one isometric symmetry with a given fixed subspace (Lemma 3.1 of [8]).

Lemma 7.1. Let $X$ be a normed space and suppose $S, T$ are two symmetries with the same fixed subspace $E$ and $\|S\|\|T\| \leq 4$. Then $\|S-T\| \leq$ $10(\log \|S\|+\log \|T\|)$.

Proof. By assumption $S(I+T)=I+T$, which implies that $T-S=$ $S T-I=S(T-S)$. Similarly, $T(T-S)=T-S$. Thus

$$
(S T)^{n+1} x-(S T)^{n}=(S T)^{n} S(T-S)=T-S, \quad n=1,2, \ldots
$$

Hence

$$
(S T)^{n}=I+n(T-S) .
$$

Pick an integer $n$ so that

$$
2 \leq\|S\|^{n}\|T\|^{n} \leq 4
$$

Then

$$
n\|T-S\| \leq 5 .
$$

Hence, since $n \geq \log 2 \cdot(\log \|S\|+\log \|T\|)^{-1}$ we have

$$
\|T-S\| \leq 10(\log \|S\|+\log \|T\|) \text {. }
$$


We will first treat the case of two-dimensional spaces where of course $\varrho_{X}=\sigma_{X}$. For real spaces the following proposition is very useful:

Proposition 7.2. Let $X$ be a two-dimensional real Banach space. Then for any $e, x \in \partial B_{X}$ there is a reflection $R$ with $\|R\| \leq \sigma_{X}$ and $R e=\|R e\| x$. Thus $\tau_{X} \leq \sigma_{X}^{2}$.

Proof. Suppose $\theta>\varrho_{X}$. Let $\mathcal{A}$ be the set of all reflections $R \in \mathcal{L}(X)$ with $\|R\|<\theta$. By Proposition 2.4 there is a continuous map $v \mapsto R_{v}$ from $\partial B_{E}$ into $\mathcal{A}$ so that $R_{v}$ fixes $v$. For fixed $v$ the set $\{R \in \mathcal{A}: R v=v\}$ is convex and so the set $\mathcal{A}$ is connected. Fix $e \in \partial B_{E}$. Let $f: \mathcal{A} \rightarrow \partial B_{E}$ be defined by $f(R)=R e /\|R e\|$. Then $f(\mathcal{A})$ is connected and since $R \in \mathcal{A}$ implies $-R \in \mathcal{A}$ the set $f(\mathcal{A})$ is symmetric about the origin. This implies $f(\mathcal{A})=\partial B_{E}$. Thus if $x \in \partial B_{E}$ we have a reflection $R^{\prime}$ with $\left\|R^{\prime}\right\|<\theta$ so that $R^{\prime} e=\left\|R^{\prime} e\right\| x$. Since this is true for every $\theta>\varrho_{X}$ it follows that there is a reflection $R$ with $\|R\| \leq \theta$ and $R e=\|R e\| x$. Thus $U e=x$ where $U=\|R e\|^{-1} R$ and $\|U\|\left\|U^{-1}\right\|=\|R\|^{2} \leq \varrho_{X}^{2}$.

For complex two-dimensional spaces we do not have a corresponding result and we will need the following:

Proposition 7.3. For any $\varepsilon>0$ there exists $\delta=\delta(\varepsilon)>0$ with the following property. Let $X$ be a two-dimensional complex Banach space with $\sigma_{X}<1+\delta$. Then there is a Euclidean norm $|\cdot|$ on $X$ such that

$$
\|x\| \leq|x| \leq(1+\varepsilon)\|x\|, \quad x \in X
$$

and for every reflection $R: X \rightarrow X$ with $\|R\|<1+\delta$ there is a reflection $R^{\prime}: X \rightarrow X$ fixing the same subspace and such that $\left|R^{\prime}\right|=1$ and $\left|R-R^{\prime}\right| \leq \varepsilon$.

Proof. Suppose this fails for some $\varepsilon>0$. Then there is a sequence of two-dimensional complex Banach spaces $X_{n}$ with $\lim _{n \rightarrow \infty} \sigma_{X_{n}}=1$ but the conclusion fails for each $X_{n}$. By selecting an appropriate basis we can suppose that $X_{n}=\left(\mathbb{C}^{2},\|\cdot\|_{n}\right)$ where

$$
|\xi| \leq\|\xi\|_{n} \leq d_{X_{n}}|\xi|
$$

and $|\cdot|$ is the usual $\ell_{2}$-norm.

By passing to a subsequence we assume that the sequence $\|\cdot\|_{n}$ converges to some norm $\|\cdot\|$ and let $X=\left(\mathbb{C}^{2},\|\cdot\|\right)$. Then $\sigma_{X}=1$ and there is an isometric reflection $R$ on every line. Now we claim there is a Hilbertian norm on $\mathbb{C}^{2}$, say $\mid \cdot l^{\prime}$, so that each such reflection is an isometry. In the case of real spaces this would follow from a result due to Auerbach (see [18, p. 408]); but it remains valid for complex spaces. Indeed, let $G$ be the group of all isometries of $X$ and define

$$
|\xi|^{\prime}=\left(\int_{S \in G}|S \xi|^{2} d \lambda(S)\right)^{1 / 2}
$$

where $\lambda$ is Haar measure on the group of isometries of $X$. 
It follows that each isometric reflection $R$ on $X$ is also an isometric reflection on $\left(\mathbb{C}^{2},|\cdot|^{\prime}\right)$; conversely, by the uniqueness of the isometric reflection on each line in $\left(\mathbb{C}^{2},|\cdot|^{\prime}\right)$, every isometric reflection on $\left(\mathbb{C}^{2},|\cdot|^{\prime}\right)$ is also an isometric reflection on $X$. Now if $|\xi|^{\prime}=|\eta|^{\prime}=1$ then there is an isometric reflection $R$ on $X$ and an $\alpha$ with $|\alpha|=1$ so that $\eta=\alpha R \xi$. From this it follows that $\|\cdot\|_{X}$ is a multiple of $|\cdot|^{\prime}$ and hence that $\lim _{n \rightarrow \infty} d_{X_{n}}=1$. But then $\|\cdot\|=|\cdot|$.

Now assume that for infinitely many $n$ there is a reflection $R_{n}: X_{n} \rightarrow X_{n}$ with $\left\|R_{n}\right\|_{n} \rightarrow 1$ but $\left\|R_{n}-R_{n}^{\prime}\right\|>\varepsilon$ where $R_{n}^{\prime}$ is the isometric reflection on $\left(\mathbb{C}^{2},|\cdot|\right)$ fixing the same subspace. Then there is a cluster point $\left(S, S^{\prime}\right)$ of the sequence $\left(R_{n}, R_{n}^{\prime}\right)$ with $\left|S-S^{\prime}\right| \geq \varepsilon$ but $S, S^{\prime}$ are isometric reflections with the same fixed subspace on $\left(\mathbb{C}^{2},|\cdot|\right)$. This contradiction proves the result.

Proposition 7.4. There exists $\varepsilon_{0}>0$ so that if $X$ is a two-dimensional complex Banach space with $\sigma_{X}<1+\varepsilon_{0}$ then there is a Hilbertian norm $|\cdot|$ (induced by an inner product $(\cdot, \cdot))$ on $X$ with the following properties:

(i) $|x| \leq\|x\| \leq 2|x|, x \in X$.

(ii) If $R$ is a reflection on $X$ with $\|R\|<1+\varepsilon_{0}$ then the isometric reflection $R^{\prime}$ on $(X,|\cdot|)$ with the same fixed subspace satisfies $\left|R-R^{\prime}\right|<1 / 200$.

(iii) If $|e|=|y|=1$ and $|(e, y)| \geq 1 / 20$ then there is a reflection $R$ on $X$ with $\|R\| \leq \sigma_{X}$ and $R e=\alpha y$ for some $\alpha \in \mathbb{C}$.

Proof. The existence of $\varepsilon_{0}$ for (i) and (ii) is given by Proposition 7.3. It remains to prove that (iii) also holds.

Fix $e \in X$ with $|e|=1$. Let $E$ be the set of all $x \in X$ with $|x|=1$ and $(e, x) \in \mathbb{R}$. The set $E$ is isometric to the two-dimensional sphere, i.e. $\partial B_{\ell_{2}^{3}(\mathbb{R})}$. If $\theta>\sigma_{X}$ then by Proposition 2.4 we can define a continuous map $x \mapsto R_{x}$ on $X \backslash\{0\}$ so that $R_{\alpha x}=R_{x}$ for $\alpha \in \mathbb{C},\left\|R_{x}\right\|<\theta$, and $R_{x}$ is a reflection fixing $x$. Similarly, we have a continuous map $x \mapsto R_{x}^{\prime}$ on $X \backslash\{0\}$ so that $R_{x}^{\prime}$ is the unique isometric reflection on $(X,|\cdot|)$ fixing $x$ and $\left\|R_{x}-R_{x}^{\prime}\right\|<1 / 100$.

Now for $x \in E$ there is a unique reflection $R_{\varphi(x)}^{\prime}$ so that $R_{\varphi(x)}^{\prime} e=x$. (The choice of $\varphi(x)$ is only unique up to scalar multiples and we do not claim $\varphi$ is continuous; however, $x \mapsto R_{\varphi(x)}^{\prime}$ is continuous.) Let us define $g(x)=R_{\varphi(x)} e$. It is easily seen that that $g: E \rightarrow X$ is a continuous function.

Note that $|g(x)-x|<1 / 100$ and $|(g(x), e)-(x, e)|<1 / 100$; thus if $|(x, e)|>1 / 100$ then $|(g(x), e)|>0$. Let $\psi$ be a continuous function on $[0, \infty)$ so that $0 \leq \psi(t) \leq 1$ and $\psi(t)=0$ for $0 \leq t<1 / 100$ and $\psi(t)=1$ if $t \geq 1 / 50$. We now define a map $h: E \rightarrow X$ by

$$
h(x)=\psi(|(e, x)|)(e, g(x))|(g(x), e)|^{-1} g(x)+(1-\psi(|(e, x)|)) x .
$$

Then $h$ is continuous and $(h(x), e)$ is real for all $x \in E$. We next observe that for any $x$ we have $h(x)=R e$, where $R$ is some reflection fixing $\varphi(x)$. 
Thus if $h(x)=\alpha x$ for some $x$ then $R_{\varphi(x)}^{\prime} R e=\alpha e$, i.e. $\alpha$ is an eigenvalue of $R_{\varphi(x)}^{\prime} R$ which is an operator with determinant one and an eigenvalue one (corresponding to the eigenvector $\varphi(x)$ ). Thus $\alpha=1$.

It now follows that we can define $f: E \rightarrow E$ by $f(x)=h(x) /|h(x)|$ and $f$ is continuous and satisfies $f(x) \neq-x$ for every $x \in E$. Thus $f$ is homotopic to the identity map. This implies, by the Borsuk antipodal mapping theorem, that $f$ is surjective (see e.g. [14, p. 29]).

Now suppose $y \in E$ and $f(x)=y$. If $|(x, e)|<1 / 50$ then since $|g(x)-x|<$ $1 / 100$ we have $|(g(x), e)|<3 / 100$. Thus $|(h(x), e)|<3 / 100$ and $|h(x)|>$ $99 / 100$. So $|(y, e)|<1 / 20$. Hence if we assume $|(y, e)| \geq 1 / 20$ we have $y=f(x)=\alpha g(x)$ for some $\alpha \in \mathbb{C}$. This implies (iii).

TheOREM 7.5. There exists a constant $0<c \leq 1$ so that if $X$ is a two-dimensional (real or complex) Banach space then

$$
c\left(d_{X}-1\right) \leq \sigma_{X}-1 \leq d_{X}-1 .
$$

Proof. The estimate $\sigma_{X} \leq d_{X}$ is trivial. We consider therefore the first inequality. The basic idea of the proof is the same in both the real and complex settings, but the details differ. Note that since $d_{X} \leq \sqrt{2}$, we have an estimate $\sigma_{X} \leq 2$ and it is only necessary to prove an estimate for $\sigma_{X}-1$ small enough.

In both cases we start by defining a map $x \mapsto R_{x}$ for $x \neq 0$ where $R_{x}$ is a reflection fixing $x$ so that $\left\|R_{x}\right\| \leq \sigma_{X}$ and $R_{\alpha x}=R_{x}$ for every scalar $\alpha$. Let $(\cdot, \cdot)$ be an inner product on $X$. In the real case this can be arbitrary, but in the complex case we assume $\sigma_{X}-1<\varepsilon_{0}$ and the inner product is chosen to satisfy Proposition 7.4. We can then write $R_{x}$ in the form

$$
R_{x} y=2\|x\|^{-2}(y, L x) x-y, \quad x, y \in X .
$$

With $L(0)=0$ this defines a map $L: X \rightarrow X$ which is homogeneous, i.e.

$$
L(\alpha x)=\alpha L(x), \quad \alpha \in \mathbb{K}, x \in X,
$$

where $\mathbb{K}=\mathbb{R}$ or $\mathbb{C}$. Note that

$$
(x, L x)=\|x\|^{2} \quad \text { and } \quad|(y, L x)| \leq \sigma_{X}\|x\|\|y\|, \quad x, y \in X .
$$

Now let $S: X \rightarrow X$ be any reflection with $\|S\| \leq \sigma_{X}$. Suppose $\|x\|=1$. Then $S R_{x} S$ is a reflection with fixed space $[S x]$ and $\left\|S R_{x} S\right\| \leq \sigma_{X}^{3}$. By Lemma 7.1,

$$
\left\|S R_{x} S-R_{S x}\right\| \leq 40 \log \sigma_{X}
$$

Thus

$$
\|S x\|(S x, L x)-\|S x\|^{-1}(x, L(S x)) \leq 20\left(\sigma_{X}-1\right) .
$$

Now

$$
\left|\|S x\|-\|S x\|^{-1}\right||(S x, L x)| \leq \sigma_{X}^{2}-1 \leq 3\left(\sigma_{X}-1\right) .
$$


Hence

$$
|(S x, L x)-(x, L S x)| \leq 23 \sigma_{X}\left(\sigma_{X}-1\right) \leq 50\left(\sigma_{X}-1\right) .
$$

Now it follows that if $x$ is arbitrary and $y=\alpha S x$ for some reflection $S$ with $\|S\| \leq \sigma_{X}$ then we have

$$
|(y, L x)-(x, L y)| \leq 100\left(\sigma_{X}-1\right)\|x\|\|y\| .
$$

Proof for real scalars. Now assume $X$ is real. We can apply Proposition 7.2 and use (7.1). We have

$$
|(y, L x)-(x, L y)| \leq 100\left(\sigma_{X}-1\right)\|x\|\|y\|, \quad x, y \in X .
$$

Suppose $x_{1}, x_{2}, y \in X$; then

$$
\begin{aligned}
& \left|\left(y, L\left(x_{1}+x_{2}\right)\right)-\left(y, L x_{1}\right)-\left(y, L x_{2}\right)\right| \\
& \quad \leq\left|\left(x_{1}+x_{2}, L y\right)-\left(x_{1}, L y\right)-\left(x_{2}, L y\right)\right|+200\left(\sigma_{X}-1\right)\left(\left\|x_{1}\right\|+\left\|x_{2}\right\|\right)\|y\|
\end{aligned}
$$

so that

$$
\left|\left(y, L\left(x_{1}+x_{2}\right)\right)-\left(y, L x_{1}\right)-\left(y, L x_{2}\right)\right| \leq 200\left(\sigma_{X}-1\right)\left(\left\|x_{1}\right\|+\left\|x_{2}\right\|\right)\|y\| .
$$

Now pick a normalized basis $e_{1}, e_{2}$ of $X$ such that $R_{e_{1}} e_{2}=-e_{2}$. Thus $\left(e_{2}, L e_{1}\right)=0$ and $\left|\left(e_{1}, L e_{2}\right)\right| \leq 100\left(\sigma_{X}-1\right)$. Then for $\xi_{1}, \xi_{2} \in \mathbb{R}$ we have

$$
\begin{aligned}
\mid \| \xi_{1} e_{1} & +\xi_{2} e_{2} \|^{2}-\xi_{1}^{2}-\xi_{2}^{2} \mid \\
& =\left|\left(\xi_{1} e_{1}+\xi_{2} e_{2}, L\left(\xi_{1} e_{1}+\xi_{2} e_{2}\right)\right)-\xi_{1}^{2}-\xi_{2}^{2}\right| \\
& =\left|\left(\xi_{1} e_{1}, L\left(\xi_{1} e_{1}+\xi_{2} e_{2}\right)\right)+\left(\xi_{2} e_{2}, L\left(\xi_{1} e_{1}+\xi_{2} e_{2}\right)\right)-\xi_{1}^{2}-\xi_{2}^{2}\right| \\
& \leq\left|\xi_{1} \xi_{2}\left(e_{1}, L e_{2}\right)\right|+400\left(\sigma_{X}-1\right)\left(\left|\xi_{1}\right|+\left|\xi_{2}\right|\right)^{2} \leq 900\left(\sigma_{X}-1\right)\left(\xi_{1}^{2}+\xi_{2}^{2}\right) .
\end{aligned}
$$

This estimate implies the result (it is necessary only to establish an estimate for $\sigma_{X}$ small enough).

Proof for complex scalars. If $X$ is complex, we recall that the inner product is chosen so that Proposition 7.4 holds. Our main task is to prove a complex analogue of (7.2). In this case we observe that if $|x|=1$ and

$$
R_{x}^{\prime} y=2(y, x) x-y, \quad y \in X,
$$

then $\left|R_{x}^{\prime}-R_{x}\right| \leq 1 / 200$ so that $\left|\|x\|^{-2} L x-x\right| \leq 1 / 400$. Thus for general $x$ we have

$$
\left.|| x\right|^{2}\|x\|^{-2} L x-x\left|\leq \frac{1}{400}\right| x \mid, \quad x \in X .
$$

Suppose $\|x\|=\|y\|=1$. Then $|(x, L y)|,|(y, L x)| \leq \sigma_{X} \leq 2$. Furthermore,

$$
R_{x} R_{y} z=4(y, L x)(z, L y) x-2(z, L y) y-2(z, L x) x+z
$$

so that

$$
\operatorname{tr} R_{x} R_{y}=4(y, L x)(x, L y)-2 .
$$


Now $\left\|R_{x} R_{y}\right\|,\left\|\left(R_{x} R_{y}\right)^{-1}\right\| \leq \sigma_{X}^{2}$ and $\operatorname{det} R_{x} R_{y}=1$. Hence the eigenvalues of $R_{x} R_{y}$ are of the form $r^{ \pm 1} e^{ \pm i \theta}$ where $\sigma_{X}^{-2} \leq r \leq \sigma_{X}^{2}$. In particular,

$$
\begin{aligned}
& \left|\operatorname{Im} \operatorname{tr} R_{x} R_{y}\right| \leq\left(\sigma_{X}^{2}-\sigma_{X}^{-2}\right) \leq 4\left(\sigma_{X}-1\right), \\
& \left|\operatorname{Retr} R_{x} R_{y}\right| \leq \sigma_{X}^{2}+\sigma_{X}^{-2} \leq 2+3\left(\sigma_{X}-1\right) .
\end{aligned}
$$

Thus

$$
\begin{aligned}
|\operatorname{Im}(y, L x)(x, L y)| & \leq\left(\sigma_{X}-1\right), \\
\operatorname{Re}(y, L x)(x, L y) & \geq-\left(\sigma_{X}-1\right) .
\end{aligned}
$$

Now suppose $|(x, L y)| \geq 1 / 3$. Then by (7.4),

$$
\left.|| y\right|^{2} L y-y\left|\leq \frac{1}{400}\right| y \mid
$$

and so

$$
|(x, y)| \geq\left(\frac{|y|}{|x|}|(x, L y)|-\frac{1}{400}\right)|x||y| .
$$

Now $|y| /|x| \geq 1 / 2$. Hence

$$
|(x, y)| \geq \frac{1}{7}|x||y|
$$

Now by Proposition 7.4 there is a reflection $S$ with $\|S\| \leq \sigma_{X}$ and $S x=\alpha y$ for complex $\alpha$. Clearly, $|\alpha|,|\alpha|^{-1} \leq \sigma_{X} \leq 2$. This implies that

$$
|\bar{\alpha}(x, L y)-\alpha(y, L x)| \leq 100\left(\sigma_{X}-1\right)|\alpha| \leq 200\left(\sigma_{X}-1\right) .
$$

Thus

$$
||(x, L y)|-|(y, L x)|| \leq 400\left(\sigma_{X}-1\right) .
$$

Hence

$$
\left.||(x, L y)\right|^{2}-|(y, L x)|^{2} \mid \leq 1600\left(\sigma_{X}-1\right) .
$$

Next we observe, using (7.6), that

$$
\begin{aligned}
|(y, L x)+\overline{(x, L y)}|^{2} & =|(y, L x)|^{2}+|(x, L y)|^{2}+2 \operatorname{Re}(y, L x)(x, L y) \\
& \geq|(x, L y)|^{2}-2\left(\sigma_{X}-1\right)>1 / 16
\end{aligned}
$$

for $\sigma_{X}-1$ small enough. Thus

$$
|(y, L x)+\overline{(x, L y)}|>1 / 4
$$

provided $\sigma_{X}-1<\varepsilon_{1}$ for some choice of $\varepsilon_{1}>0$. Now

$$
\begin{aligned}
& (\overline{(y, L x)}-(x, L y))((y, L x)+\overline{(x, L y)}) \\
& =|(y, L x)|^{2}-|(x, L y)|^{2}-2 \operatorname{Im}(x, L y)(y, L x)
\end{aligned}
$$

so that, by (7.5),

$$
|\overline{(y, L x)}-(x, L y)| \leq 10^{4}\left(\sigma_{X}-1\right) .
$$

Notice the same estimate holds if $|(y, L x)| \geq 1 / 3$. 
It follows for arbitrary $x, y$ that if either $|(x, L y)| \geq \frac{1}{3}\|x\|\|y\|$ or $|(y, L x)|$ $\geq \frac{1}{3}\|x\|\|y\|$ we have

$$
|\overline{(y, L x)}-(x, L y)| \leq 10^{4}\left(\sigma_{X}-1\right)\|x\|\|y\| .
$$

Now let us assume $\|x\|=\|y\|=1$ and $|(x, L y)|,|(y, L x)|<1 / 3$. Then if $|\alpha|=1$, we have $|(x+\alpha y, L y)|>2 / 3 \geq \frac{1}{3}\|x+\alpha y\|\|y\|$ and $|(x+\alpha y, L x)| \geq$ $\frac{1}{3}\|x+\alpha y\|\|x\|$. Now

$$
\|x+\alpha y\|^{2}=(x+\alpha y, L(x+\alpha y)=(x, L(x+\alpha y))+\alpha(y, L(x+\alpha y)) .
$$

By (7.7),

$$
\begin{aligned}
|(x, L(x+\alpha y))-\overline{(x+\alpha y, L x)}| & \leq 2 \cdot 10^{4}\left(\sigma_{X}-1\right), \\
|(y, L(x+\alpha y))-\overline{(x+\alpha y, L y)}| & \leq 2 \cdot 10^{4}\left(\sigma_{X}-1\right) .
\end{aligned}
$$

Hence, whenever $|\alpha|=1$,

$$
|\operatorname{Im}(\bar{\alpha}(y, L x)+\alpha(x, L y))| \leq 4 \cdot 10^{4}\left(\sigma_{X}-1\right),
$$

which implies

$$
|\overline{(y, L x)}-(x, L y)| \leq 4 \cdot 10^{4}\left(\sigma_{X}-1\right) .
$$

Thus we obtain the estimate

$$
|\overline{(y, L x)}-(x, L y)| \leq 4 \cdot 10^{4}\left(\sigma_{X}-1\right)\|x\|\|y\| .
$$

This is the complex analogue of (7.2) and the remainder of the proof is very similar to the real case, albeit with much larger constants.

We now use this characterization to give an estimate for $\varrho_{X}$.

THEOREM 7.6. There are constants $0<c<C<\infty$ such that for every (real or complex) Banach space $X$,

$$
c\left(d_{2}(X)-1\right) \leq \varrho_{X}-1<C\left(d_{2}(X)-1\right) .
$$

Proof. The first inequality follows from Theorem 7.5.

Now suppose that $d_{2}=d_{2}(X) \leq 4 / 3<\sqrt{2}$. Then there exists an absolute choice of $1<p<2$ and $C$ so that $T_{p}(X) \leq C$ (Proposition 2.3). Furthermore, by Proposition 2.2 we have $d_{4}(X) \leq d_{2}^{8}$.

Fix $x \in \partial B_{X}$. For every two-dimensional subspace $E$ containing $x$ there is an inner product $(\cdot, \cdot)_{E}$ inducing a Euclidean norm $|\cdot|_{E}$ on $E$ with $|y|_{E} \leq$ $\|y\| \leq d_{2}|y|_{E}$ for $y \in E$.

Let us define a functional $\varphi: X \rightarrow \mathbb{K}$ (with $\mathbb{K}=\mathbb{R}$ or $\mathbb{C}$ ) by

$$
\varphi(y)=(y, x)_{[x, y]}
$$

where $x, y$ are linearly independent, and

$$
\varphi(\alpha x)=\alpha, \quad \alpha \in \mathbb{K} .
$$

Note that $\varphi$ is homogeneous, i.e. $\varphi(\alpha y)=\alpha \varphi(y)$ for $\alpha \in \mathbb{K}$. 
Next suppose $y_{1}, y_{2}, y_{3} \in X$ with $y_{1}+y_{2}+y_{3}=0$. Let $F$ be the (threedimensional) linear span of $x, y_{1}, y_{2}, y_{3}$. Then there is an inner-product norm $|\cdot|_{F}$ on $F$ with

$$
\|v\| \leq|v|_{F} \leq d_{2}^{8}\|v\|, \quad v \in F .
$$

Let $E_{j}=\left[x, y_{j}\right]$ for $j=1,2,3$. Then we can write

$$
(v, w)_{E_{j}}=\left(v, A_{j} w\right)_{F}
$$

where $A_{j}$ is a positive operator on $\left(E_{j},|\cdot|_{F}\right)$ with $I \leq A_{j} \leq d_{2}^{10} I$. Thus

$$
\begin{aligned}
\left|\sum_{j=1}^{3} \varphi\left(y_{j}\right)\right| & =\left|\sum_{j=1}^{3}\left(y_{j}, A_{j} x\right)_{F}\right|=\left|\sum_{j=1}^{3}\left(y_{j},\left(A_{j}-I\right) x\right)_{F}\right| \\
& \leq\left(d_{2}^{10}-1\right) \sum_{j=1}^{3}|x|_{F}\left|y_{j}\right|_{F} \leq d_{2}^{16}\left(d_{2}^{10}-1\right) \sum_{j=1}^{3}\left\|y_{j}\right\| .
\end{aligned}
$$

In view of the uniform estimate on $T_{p}(X)$ this implies by results of [12] or [5] that we can find a linear map $x^{*} \in X^{*}$ so that

$$
\left|\varphi(y)-x^{*}(y)\right| \leq C_{2}\left(d_{2}-1\right)\|y\|
$$

where $C_{2}$ is an absolute constant. We can suppose that $x^{*}(x)=\varphi(x)=1$. Let us remark that the papers cited refer to the real case, but the complex case can be treated similarly by approximating $\operatorname{Re} \varphi$ by a real linear functional $x_{r}^{*}$ and then setting $x^{*}(y)=x_{r}^{*}(y)-i x_{r}^{*}(i y)$.

Now for any $y \in X$ we have

$$
|2 \varphi(y) x-y|_{[x, y]} \leq|y|_{[x, y]} \leq\|y\|
$$

so that

$$
\|2 \varphi(y) x-y\| \leq d_{2}\|y\| .
$$

Then the reflection $R y=2 x^{*}(y) x-y$ satisfies

$$
\|R y\| \leq\left(C_{2}\left(d_{2}-1\right)+d_{2}\right)\|y\|, \quad y \in X .
$$

This gives the required estimate.

REMARK. If $\varrho_{X}=1$, so that every one-dimensional subspace is the fixed space of an isometric symmetry, then $d_{2}(X)=1$, which implies that $d_{X}=1$, (using the Jordan-von Neumann theorem [9]). Note that this applies even for spaces of dimension two.

In what follows we will need only one special case of this result, which we state explicitly for future reference. We remark that our proof only relies on the real version of Theorem 7.5, which is much easier than the complex version. 
Lemma 7.7. There is a constant $C$ so that if $X=\mathbb{R} \oplus_{N} \mathbb{R}$ or $X=\mathbb{C} \oplus_{N} \mathbb{C}$ for some normalized absolute norm on $\mathbb{R}^{2}$ and $\sigma_{X}=1+\varepsilon$ then

$$
(1+C \varepsilon)^{-1}\left(\left|\xi_{1}\right|^{2}+\left|\xi_{2}\right|^{2}\right)^{1 / 2} \leq N\left(\xi_{1}, \xi_{2}\right) \leq(1+C \varepsilon)\left(\left|\xi_{1}\right|^{2}+\left|\xi_{2}\right|^{2}\right)^{1 / 2}, \quad \xi_{1}, \xi_{2} \in \mathbb{R} .
$$

Proof. We first reduce to the real case. Indeed, suppose $X=\mathbb{C} \oplus_{N} \mathbb{C}$ and $Y=\mathbb{R} \oplus_{N} \mathbb{R}$. We claim that $\sigma_{Y} \leq \sigma_{X}$. For suppose $y \in Y$. Then there is a rank-one projection $P: X \rightarrow \mathbb{C} y$ with $\|I-2 P\| \leq \sigma_{X}$. Then $P$ is represented by a complex $2 \times 2$ matrix. However, since $y \in Y$ the matrix obtained by taking the real part of each entry is also a rank-one projection $Q$ onto $\mathbb{C} y$ and $\|I-2 Q\| \leq\|I-2 P\|$. Now $Q$ restricts to a projection on $Y$.

Thus we may suppose $X=\mathbb{R} \oplus_{N} \mathbb{R}$. Let $d=d_{X}$. Then there is a Euclidean norm $|\cdot|$ on $X$ so that $\|x\| \leq|x| \leq d\|x\|$ for $x \in X$. Thus

$$
\begin{aligned}
\left\|\xi_{1} e_{1}+\xi_{2} e_{2}\right\|^{2} & \leq \frac{1}{2}\left(\left|\xi_{1} e_{1}+\xi_{2} e_{2}\right|^{2}+\left|\xi_{1} e_{1}-\xi_{2} e_{2}\right|^{2}\right)=\left|\xi_{1}\right|^{2}\left|e_{1}\right|^{2}+\left|\xi_{2}\right|^{2}\left|e_{2}\right|^{2} \\
& \leq d^{2}\left(\left|\xi_{1}\right|^{2}+\left|\xi_{2}\right|^{2}\right)
\end{aligned}
$$

and

$$
\begin{aligned}
\left|\xi_{1}\right|^{2}+\left|\xi_{2}\right|^{2} & \leq\left|\xi_{1}\right|^{2}\left|e_{1}\right|^{2}+\left|\xi_{2}\right|^{2}\left|e_{2}\right|^{2}=\frac{1}{2}\left(\left|\xi_{1} e_{1}+\xi_{2} e_{2}\right|^{2}+\left|\xi_{1} e_{1}-\xi_{2} e_{2}\right|^{2}\right) \\
& \leq d^{2}\left\|\xi_{1} e_{1}+\xi_{2} e_{2}\right\|^{2} .
\end{aligned}
$$

Now using Theorem 7.6 completes the proof.

Proposition 7.8. There is an absolute constant $C$ so that if $X$ is a finite-dimensional real or complex normed space and $Y$ is a Hilbert enlargement of $X$ with $\sigma_{Y}<2$ then

$$
d_{X}-1 \leq C\left(\sigma_{Y}-1\right) .
$$

Proof. Let $Y=X \oplus H$ and $\sigma_{Y}=1+\varepsilon$.

Assume $e \in X$ and $h \in H$ with $\|e\|=\|h\|=1$. Then let $E=[e, h]$ and note that $\sigma_{E} \leq 1+\varepsilon$. Note also that for some normalized absolute norm, we have

$$
\left\|\xi_{1} e+\xi_{2} h\right\|=N\left(\left|\xi_{1}\right|,\left|\xi_{2}\right|\right) .
$$

It now follows from Lemma 7.7 that if $Z$ is the $\ell_{2}$-sum $X \oplus_{2} H$ then $d(Y, Z)$ $\leq 1+C_{1} \varepsilon$ for some constant $C_{1}$ and thus $\sigma_{Z} \leq 1+C_{2} \varepsilon$ for some other constant $C_{2}$. We will now work on $Z$.

Now for $\varepsilon<1$ we have an estimate $d_{X} \leq M$ where $M$ is independent of $\varepsilon$. In particular, there is an isomorphism $U: X \rightarrow H$ with $\|U\|=1$ and $\left\|U^{-1}\right\| \leq M$. Let $G=(I+U) X \subset Z$. Then there is a projection $P: Z \rightarrow G$ with $\|I-2 P\| \leq 1+C_{2} \varepsilon$. Let $F=(I-P) Z$ be the complementary subspace. Suppose $e+h \in F$ where $e \in X$ and $h \in H$. Let $h=U x$. We will show that if $\varepsilon$ is small enough we have an upper bound on $\|h\|$. Note that

$$
\|e+x+2 h\| \leq\left(1+C_{2} \varepsilon\right)\|e-x\|
$$


so that

$$
4\|h\|^{2} \leq\left(1+C_{2} \varepsilon\right)^{2}\|e-x\|^{2}-\|e+x\|^{2} .
$$

Hence, using $\|x\| \leq M\|h\|$, we have

$$
\begin{aligned}
4\|h\|^{2} & \leq\left(\|e-x\|^{2}-\|e+x\|^{2}\right)+C_{2} \varepsilon\left(2+C_{2} \varepsilon\right)\|e-x\|^{2} \\
& \leq 4\|e\|\|x\|+C_{2} \varepsilon\left(2+C_{2} \varepsilon\right)(\|e\|+\|x\|)^{2} \\
& \leq 8 M^{2}\|e\|^{2}+\frac{1}{2} M^{-2}\|x\|^{2}+2 C_{2} \varepsilon\left(2+C_{2} \varepsilon\right)\left(\|e\|^{2}+\|x\|^{2}\right) \\
& \leq\left(8 M^{2}+2 C_{2} \varepsilon\left(2+C_{2} \varepsilon\right)\right)\|e\|^{2}+\left(\frac{1}{2}+2 M^{2} C_{2} \varepsilon\left(2+C_{2} \varepsilon\right)\right)\|h\|^{2} .
\end{aligned}
$$

Provided $\varepsilon$ is small enough, say $0<\varepsilon \leq \varepsilon_{2}$, we therefore get a bound

$$
\|h\| \leq M^{\prime}\|e\|
$$

for some absolute constant $M^{\prime}$ independent of $\varepsilon$. Under these hypotheses we can write $F=(I-V) X$ for a suitable operator $V: X \rightarrow H$ with $\|V\| \leq M^{\prime}$.

Now suppose $x \in E$. Then $\|2 x+(U-V) x\|,\|U x+V x\| \leq\left(M^{\prime}+3\right)\|x\|$ and so

$$
|\|2 x+(U-V) x\|-\|U x+V x\|| \leq C_{2}\left(M^{\prime}+3\right) \varepsilon\|x\|
$$

or

$$
\left|\|2 x+(U-V) x\|^{2}-\|U x+V x\|^{2}\right| \leq 2 C_{2}\left(M^{\prime}+3\right)^{2} \varepsilon\|x\|^{2} .
$$

This reduces to

$$
4\left|\|x\|^{2}-(U x, V x)\right| \leq 2 C_{2}\left(M^{\prime}+3\right)^{2} \varepsilon\|x\|^{2} .
$$

It follows that if we define $|x|^{2}=(U x, V x)$ for $x \in X$ we have

$$
\left.4\left|\|x\|^{2}-\right| x\right|^{2} \mid \leq 2 C_{2}\left(M^{\prime}+3\right)^{2} \varepsilon\|x\|^{2} .
$$

This implies an estimate

$$
d_{X} \leq 1+C_{3} \varepsilon
$$

if $0<\varepsilon<\varepsilon_{3}$ for some suitable $\varepsilon_{3}>0$.

TheOREM 7.9. There exists a constant $0<C<\infty$ so that if $X$ is an infinite-dimensional (real or complex) Banach space and $1 \leq \sigma_{X} \leq 2$ then

$$
\sigma_{X}-1 \leq d_{X}-1 \leq C\left(\sigma_{X}-1\right)
$$

Proof. It is clear that $\sigma_{X} \leq d_{X}$. The other inequality is an immediate conclusion from Propositions 4.2 and 7.8 .

\section{The finite-dimensional problem}

THEOREm 8.1. (i) Suppose $0<a<1 / 3$. Then there is a constant $C=$ $C(a)$ so that if $X$ is an $n$-dimensional Banach space with $\lambda_{X}<2$ and $n \geq 3$ we have

$$
d_{X}-1 \leq C\left(\sqrt{\lambda_{X}-1}+n^{-a}\right)
$$


(ii) Suppose $0<a<1$. Then there is a constant $C=C(a)$ so that if $X$ is an $n$-dimensional Banach space with $\sigma_{X}<2$ we have

$$
d_{X}-1 \leq C\left(\left(\sigma_{X}-1\right)+n^{-a}\right) .
$$

Proof. Let $0<\theta<1$ and $E$ be a subspace of $X$ with $\operatorname{dim} E=m \leq n^{\theta}$. In view of Proposition 2.2 (or see [7]) we can bound $d_{X} \leq M$ for some $M$ and thus if $n$ is large enough we can apply Proposition 4.3 and hence there exists a Hilbert enlargement $Y$ of $E$ with $\operatorname{dim} Y=2 m$ and

$$
d\left(Y, Y_{0}\right) \leq 1+40 M \sqrt{\frac{m}{n} \log \frac{n}{m}}
$$

for some subspace $Y_{0}$ of $X$. Hence in (i),

$$
\lambda_{Y} \leq d\left(Y, Y_{0}\right) \lambda_{X}
$$

This leads to an estimate

$$
\lambda_{Y}-1 \leq C_{0}\left(n^{(\theta-1) / 2}+\lambda_{X}-1\right), \quad n \geq n_{0}, \lambda_{X}<2,
$$

where $C_{0}=C_{0}(\theta)$. Hence by Proposition 6.1 we have

$$
d_{E}-1 \leq C_{1}\left(n^{(\theta-1) / 4}+\sqrt{\lambda_{X}-1}\right) .
$$

Thus

$$
d_{m}(X) \leq C_{1}\left(n^{(\theta-1) / 4}+\sqrt{\lambda_{X}-1}\right) .
$$

This implies an estimate (for $n$ large enough) using Proposition 2.2,

$$
d_{X}-1 \leq C_{2}\left(n^{(\theta-1) / 4}+\sqrt{\lambda_{X}-1}\right) .
$$

In particular, there exist $\varepsilon_{0}>0$ and $n_{1}$ so that $d_{X}<1.04$ provided $n \geq n_{1}$ and $\lambda_{X}<1+\varepsilon_{0}$. In this case we can revise our estimate on $d\left(Y, Y_{0}\right)$ to give

$$
d\left(Y, Y_{0}\right) \leq 1+200 \sqrt{d_{X}-1} \sqrt{\frac{m}{n} \log \frac{n}{m}}
$$

and so

$$
\lambda_{Y}-1 \leq C_{3}\left(n^{(\theta-1) / 2} \sqrt{d_{X}-1}+\left(\lambda_{X}-1\right)\right), \quad n \geq n_{1}, \lambda_{X}<1+\varepsilon_{0},
$$

which implies

$$
d_{X}-1 \leq C_{4}\left(\left(d_{X}-1\right)^{1 / 4} n^{(\theta-1) / 4}+\sqrt{\lambda_{X}-1}\right) .
$$

This in turn implies

$$
d_{X}-1 \leq C_{5}\left(n^{(\theta-1) / 3}+\sqrt{\lambda_{X}-1}\right), \quad n \geq n_{1}, \lambda_{X}<1+\varepsilon_{0},
$$

and a similar estimate with a different constant must hold if $n \geq 3$ and $\lambda_{X}<2$. This proves (i).

(ii) is similar. In this case one gets

$$
d_{X}-1 \leq C_{6}\left(n^{(\theta-1) / 2} \sqrt{d_{X}-1}+\left(\sigma_{X}-1\right)\right), \quad n \geq n_{2}, \sigma_{X}<1+\varepsilon_{1} .
$$


This implies

$$
d_{X}-1 \leq C_{7}\left(n^{\theta-1}+\left(\sigma_{X}-1\right)\right), \quad n \geq n_{2}, \sigma_{X}<1+\varepsilon_{1} .
$$

TheOREM 8.2. There is a constant $C$ so that if $X$ is a finite-dimensional normed space with $\sigma_{X}<2$ we have

$$
d_{X}-1 \leq C\left(\sigma_{X}-1\right)\left|\log \left(\sigma_{X}-1\right)\right| .
$$

Proof. Let $\operatorname{dim} X=n$. Assume first that $\sigma_{X}-1>n^{-1 / 2}$. Then we have an estimate $d_{X}-1 \leq C\left(\sigma_{X}-1\right)$ by Theorem 8.1. If $\sigma_{X}-1 \leq n^{-1 / 2}$ we have an estimate $\log d_{2}(X) \leq C\left(\sigma_{X}-1\right)$ by Theorem 7.6. Hence (see [7])

$$
\log d_{X} \leq C_{0}\left(\sigma_{X}-1\right) \log n \leq C_{1}\left(\sigma_{X}-1\right)\left|\log \left(\sigma_{X}-1\right)\right| .
$$

TheOrem 8.3. There is a constant $C$ so that if $X$ is any Banach space with $\lambda_{X}<2$ we have

$$
\sigma_{X}-1 \leq C\left(\sqrt{d_{2}(X)-1}+\sqrt{\lambda_{X}-1}\right) .
$$

Proof. Let $E$ be a finite-dimensional subspace of $X$ and $P$ be a projection of $X$ onto $E$ with $\|P\| \leq \lambda_{X}$. Let $F=[P x, x]$. Then there is a Euclidean norm $|\cdot|$ on $F$ with $\|y\| \leq|y| \leq d_{2}(X)\|y\|$ for $y \in F$. Thus the norm of the restriction $P_{F}$ of $P$ to $(F,|\cdot|)$ can be estimated by $\left|P_{F}\right| \leq d_{2}(X) \lambda_{X}$. Since $P_{F}$ is a rank-one projection there is a rank-one orthogonal projection $Q$ with $\left|P_{F}-Q\right| \leq \sqrt{d_{2}(X) \lambda_{X}-1}$ and so

$$
\left|1-2 P_{F}\right| \leq 1+2 \sqrt{d_{2}(X) \lambda_{X}-1} .
$$

Thus

$$
\|(I-2 P) x\| \leq d_{2}(X)\left(1+2 \sqrt{d_{2}(X) \lambda_{X}-1}\right)\|x\|, \quad x \in X .
$$

TheOREM 8.4. Suppose $0<a<1$. Then there is a constant $C=C(a)$ so that if $X$ is a finite-dimensional Banach space with $\operatorname{dim} X \geq 3$ and $\lambda_{X}<2$ we have

$$
d_{X}-1 \leq C\left(\left(\varphi_{3}\left(\lambda_{X}\right)-1\right)^{a / 2}+\left(\lambda_{X}-1\right)^{a / 2}\right) .
$$

Hence

$$
\lim _{\lambda \rightarrow 1} \varphi_{\infty}(\lambda)=1
$$

Proof. We have $d_{2}(X) \leq d_{3}(X) \leq \varphi_{3}\left(\lambda_{X}\right)$. The theorem then follows from Theorems 8.3 and 8.2 .

\section{References}

[1] F. Albiac and N. J. Kalton, Topics in Banach Space Theory, Grad. Texts in Math. 233, Springer, New York, 2006.

[2] Y. Benyamini and J. Lindenstrauss, Geometric Nonlinear Functional Analysis. Vol. 1, Amer. Math. Soc. Colloq. Publ. 48, Amer. Math. Soc., Providence, RI, 2000. 
[3] W. Blaschke, Kreis und Kugel, Veit, Leipzig, 1916, reprinted by Chelsea, New York, 1949.

[4] F. Bohnenblust, A characterization of complex Hilbert spaces, Portugal. Math. 3 (1942), 103-109.

[5] Y. A. Brudnyi and N. J. Kalton, Polynomial approximation on convex subsets of $\mathbf{R}^{n}$, Constr. Approx. 16 (2000), 161-199.

[6] T. Figiel, Review of [10], Math. Rev. 53 (1977), \#3649.

[7] T. Figiel, J. Lindenstrauss, and V. D. Milman, The dimension of almost spherical sections of convex bodies, Acta Math. 139 (1977), 53-94.

[8] G. Godefroy, N. J. Kalton, and P. D. Saphar, Unconditional ideals in Banach spaces, Studia Math. 104 (1993), 13-59.

[9] P. Jordan and J. von Neumann, On inner products in linear metric spaces, Ann. of Math. (2) 36 (1935), 719-723.

[10] M. I. Kadec and B. S. Mitjagin [M. I. Kadets and B. S. Mityagin], Complemented subspaces in Banach spaces, Uspekhi Mat. Nauk 28 (1973), no. 6, 77-94 (in Russian); English transl.: Russian Math. Surveys 28 (1973), no. 6, 77-95.

[11] S. Kakutani, Some characterizations of Euclidean space, Japan. J. Math. 16 (1939), 93-97.

[12] N. J. Kalton, The three space problem for locally bounded F-spaces, Compos. Math. 37 (1978), 243-276.

[13] J. Lindenstrauss and L. Tzafriri, On the complemented subspaces problem, Israel J. Math. 9 (1971), 263-269.

[14] J. Matoušek, Using the Borsuk-Ulam Theorem, Universitext, Springer, Berlin, 2003.

[15] V. D. Milman and G. Schechtman, Asymptotic Theory of Finite-Dimensional Normed Spaces, Lecture Notes in Math. 1200, Springer, Berlin, 1986.

[16] V. P. Odinec, On a property of reflexive Banach spaces with a transitive norm, Bull. Acad. Polon. Sci. Sér. Sci. Math. 30 (1982), 353-357.

[17] B. Randrianantoanina, A note on the Banach-Mazur problem, Glasgow Math. J. 44 (2002), 159-165.

[18] S. Rolewicz, Metric Linear Spaces, 2nd ed., Math. Appl. (East Eur. Ser.) 20, PWN, Warszawa, and Reidel, Dordrecht, 1985.

[19] A. Skorik and M. Zaidenberg, On isometric reflections in Banach spaces, Mat. Fiz. Anal. Geom. 4 (1997), 212-247.

[20] A. Zygmund, Trigonometric Series. Vols. I, II, 2nd ed., Cambridge Univ. Press, New York, 1959.

Department of Mathematics

University of Missouri-Columbia

Columbia, MO 65211, U.S.A.

E-mail: nigel@math.missouri.edu

Received January 9, 2007

Revised version June 11, 2008

$(6045)$ 